\title{
Regularity properties for Monge transport density and for solutions of some shape optimization problem
}

Received: 16 November 2000 / Accepted: 4 December 2000 /

Published online: 18 January 2002 - (c) Springer-Verlag 2002

\begin{abstract}
In this paper we study the dimension of some measures which are related to the classical Monge's optimal mass transport problem and are solutions of a scalar shape optimization problem. Moreover in the case of maximal dimension we will study the summability of the associate densities.
\end{abstract}

\section{Introduction}

In this introductory section we briefly describe the Monge-Kantorovich problem, the shape optimization problem and the connections between them.

\subsection{The Monge-Kantorovich problem}

This problem can be formulated in a very general setting, hence in this section $M$ will be a metric space equipped with a distance $d$. In the rest of the paper, however, the ambient space will be an open, bounded and convex subset $\Omega$ of $\mathbb{R}^{N}$ equipped with the euclidean distance.

Given two positive measures $f^{+}, f^{-}$on $M$ of equal total mass, the transport problem consists in finding, in the set of measurable maps $\varphi: M \rightarrow M$ such that $\varphi_{\#} f^{+}=f^{-}$(where $\varphi_{\#}$ is the push-forward of any measurable mapping $\varphi: M \rightarrow M)$, the minimum of the "work" functional

$$
\int_{M} d(x, \varphi(x)) d f^{+}(x)
$$

where $d$ is the distance on $M$. Each of the admissible maps $\varphi$ can be thought as a way to transport $f^{+}$on $f^{-}$and then will be called a transport. The set of such transports can be empty, as it happens for example for $f^{+}=\delta_{0}$ and $f^{-}=\left(\delta_{1}+\delta_{-1}\right) / 2$. A weak formulation of Monge's problem is the following:

$$
\min \left\{\int_{M \times M} d(x, y) d \gamma(x, y): \gamma \in \mathcal{M}^{+}(M \times M), \pi_{\#}^{1} \gamma=f^{+}, \pi_{\#}^{2} \gamma=f^{-}\right\} .
$$

L. De Pascale: Dipartimento di Matematica Applicata, Università di Pisa, via Bonanno Pisano 25/B, 56127 Pisa, Italy (e-mail: depascal@dm.unipi.it)

A. Pratelli: Scuola Normale Superiore, Piazza dei Cavalieri 7, 56127 Pisa, Italy

(e-mail:pratelli@cibs.sns.it) 
The set of the measures $\gamma$ admissible for (1.2), we will call each of them a plan of transport, is not empty as it always contains at least $f^{+} \otimes f^{-}$. It is a standard fact that if $M$ is compact (1.2) has solution.

To each transport $\varphi$ one can associate the plan of transport $\gamma_{\varphi}:=(i d \times \varphi)_{\#} f^{+}$, and $\int_{M \times M} d(x, y) d \gamma_{\varphi}(x, y)=\int_{M} d(x, \varphi(x)) d f^{+}(x)$, then the minimum in (1.2) is smaller than the infimum of (1.1). If the ambient space is an open, bounded and convex subset $\Omega$ of $\mathbb{R}^{N}$ and $f^{+}$has no atom the equality holds (see [14] and [1]), but in general the strict inequality can hold: this happens obviously when the set of transports is empty, but also in less trivial cases.

An optimal map $\varphi$ for problem (1.1) will be called an optimal transport, while an optimal measure $\gamma$ for problem (1.2) will be called an optimal plan of transport because $(x, y) \in \operatorname{spt}(\gamma)$ means, in some sense, that part of the mass in $x$ should be moved in $y$ in order to minimize the work.

\subsection{The shape optimization problem}

Let $\Omega$ be an open, bounded subset of $\mathbb{R}^{N}$ and assume that we are given an heat source $f$ and a certain amount $m$ of a conductor $C$ (i.e. a material with a positive conductivity coefficient). What is the best way to distribute $C$ in the assigned region $\Omega$ ? The optimality criterion we will accept is that of the minimal "compliance". Taking as a model for the distribution of $C$ a nonnegative bounded measure $\mu$ in $\Omega$ such that $\int_{\Omega} d \mu=m$, the energy associated to a smooth distribution of temperature $u \in \mathcal{D}(\Omega)$ is given by:

$$
E(\mu, u)=\frac{1}{2} \int_{\Omega}|D u(x)|^{2} d \mu-\langle f, u\rangle .
$$

For an assigned distribution $\mu$ of material let us define the following quantity

$$
\mathfrak{C}(\mu):=\inf _{u \in \mathcal{D}(\Omega)} E(\mu, u)
$$

the quantity $-\mathfrak{C}(\mu)$ is usually called compliance. There exists an equilibrium temperature $u_{\mu}$ (not necessarily smooth) which realizes $\min _{u} \bar{E}(\mu, u)=\mathfrak{C}(\mu)$, where $\bar{E}$ is the relaxed energy of (1.3) that we will write explicitely in (2.2). A distribution $\mu_{1}$ of material is better than $\mu_{2}$ if $\mathfrak{C}\left(\mu_{1}\right)>\mathfrak{C}\left(\mu_{2}\right)$, therefore it is natural to look for the maximum of $\mathfrak{C}(\mu)$. It turns out (and we will see it in Sect. 3) that the problem

$$
\mathcal{E}(f):=\sup \left\{\mathfrak{C}(\mu): \mu \in \mathcal{M}^{+}(\Omega), \int d \mu=m\right\}
$$

is related to

by the formula

$$
I(f):=\inf \left\{\langle-f, u\rangle: u \in \operatorname{Lip}_{1}(\Omega)\right\}
$$

$$
\mathcal{E}(f)=-\frac{I(f)^{2}}{2 m}
$$

and (1.5) is dual to

$$
\sup \left\{-\int d|\nu|: \nu \in \mathcal{M}\left(\Omega, \mathbb{R}^{N}\right),-\operatorname{div} \nu=f\right\}
$$

formula (1.6) was proved in [5], while the duality argument is standard. 
It is possible to prove (see [5]) that these problems are related to

$$
(W M K)= \begin{cases}-\operatorname{div}\left(\mu D_{\mu} u\right)=f & \text { on } \Omega \\ \left|D_{\mu} u\right|=1 & \mu-a . e .,\end{cases}
$$

where $D_{\mu} u$ is the tangential gradient of $u$ with respect to $\mu$ introduced in [4] whose definition and main properties will be recalled in Sect. 2.1.

More precisely, as proved in [5] and in Sect. 3 of this paper, the relationship between the different problems is given by the fact that if $u$ and $\nu$ are extremals for (1.5) and (1.7) respectively, then $(u,|\nu|)$ solves (3.1). On the other hand, if $(u, \mu)$ solves (3.1) then $u$ and $\mu D_{\mu} u$ are extremals for (1.5) and (1.7) respectively. Finally $\mu$ solves (3.1) together with some $u \in \operatorname{Lip}_{1}(\Omega)$ if and only if $-m \mu / I(f)$ solves (1.4).

Problem (1.4) is usually referred to as shape optimization problem and a measure which realizes the sup is called optimal shape. However, since the measures $\mu$ in the solutions of (3.1) are, up to a constant, optimal for (1.4), in this paper we will refer to these measure -and not to the solutions of (1.4)- as optimal shapes.

\subsection{Optimal plans and transport densities}

In Sect. 4 we will give the following formula that associates to each optimal plan of transport $\gamma$ an optimal shape $\mu$, where $f=f^{+}-f^{-}$:

$$
\langle\mu, \varphi\rangle:=\int_{\Omega \times \Omega}\left(\int_{\Omega} \varphi(z) d \mathcal{H}_{x y}^{1}(z)\right) d \gamma(x, y) ;
$$

moreover each optimal shape can be obtained by an optimal measure $\gamma$ in this way, as proved in [1]. In this setting $\mu$ is called transport density.

The support of an optimal plan of transport $\gamma$ enjoys a very important property called $d$-cyclical monotonicity. This property has been widely used (see for example [15]) in the case of Monge-Kantorovich problems with strictly convex costs (i.e. when $d$ in (1.1) is replaced by a strictly convex, positive function of $d$ ) and much less in the case of linear costs, as in this paper and in [6]. In fact we will use this property of $\gamma$ to deduce some relationship between the dimension or the summability of $f^{+}$and $f^{-}$and the related properties of the transport density $\mu$ (the concept of the dimension of a measure will be recalled in Sect. 2.2). Let us be more precise about the notion of cyclical monotonicity:

Definition 1.1. A set $S \subseteq M \times M$ is said d-cyclically monotone (or simply cyclically monotone if $d$ is the euclidean distance in a subset of $\mathbb{R}^{N}$ ) if for any integer $n$, any set of pairs $\left(x_{1}, y_{1}\right), \ldots,\left(x_{n}, y_{n}\right) \in S$ and any permutation $\sigma$ in $\mathfrak{S}_{n}$

$$
d\left(x_{1}, y_{1}\right)+\cdots+d\left(x_{n}, y_{n}\right) \leq d\left(x_{1}, y_{\sigma(1)}\right)+\cdots+d\left(x_{n}, y_{\sigma(n)}\right) .
$$

In the case $n=2,(1.8)$ becomes

$$
d\left(x_{1}, y_{1}\right)+d\left(x_{2}, y_{2}\right) \leq d\left(x_{1}, y_{2}\right)+d\left(x_{2}, y_{1}\right),
$$

which holds whenever $\left(x_{1}, y_{1}\right)$ and $\left(x_{2}, y_{2}\right)$ belong to $S$. Even if the general property (1.8) is stronger and it is not difficult to construct an example for which (1.9) holds but (1.8) does not, the proofs of Sect. 4 will use only the second and the representation of $\mu$ given in (4.2). 
Our interest about the notion of the cyclical monotonicity follows by the well known fact (see for example [15]) that if $\gamma$ is an optimal plan of transport then $\operatorname{spt}(\gamma)$ is $d$-cyclically monotone. Hence we will use (1.9) for $\left(x_{1}, y_{1}\right)$ and $\left(x_{2}, y_{2}\right)$ in $\operatorname{spt}(\gamma)$.

Some of the main results of the paper are the following

Theorem If $f^{+}$(or $\left.f^{-}\right)$is absolutely continuous then any transport density is also absolutely continuous. If both $f^{+}$and $f^{-}$are in $L^{\infty}$ then any transport density is in $L^{\infty}$. If both $f^{+}$and $f^{-}$are in $L^{q}$ then any transport density is in $\bigcap_{p<q} L^{p}$.

The plan of the paper is the following: in the next section we will recall some notations and some results we will use in the paper. In Sect. 3 we will study some properties of the solutions of (3.1) and prove the connections between problems (3.1), (1.4), (1.5) and (1.7). In Sect. 4, after some technical lemmas, we will prove a lower estimate on the dimension (in the sense of Sect. 2.2) of a generic transport density and we will show that no nontrivial upper estimate can be given, finally we will discuss the summability properties. Examples on the sharpness of the estimates will be given in each case.

Note. After the completion of this work we heard about a related work by Feldman and McCann, [12]. In $\mathbb{R}^{N}$ equipped with a uniformly strictly convex norm, they study the case of the transport densities related to optimal transports and, under the assumption that $f^{+}$and $f^{-}$are absolutely continuous, they prove the absolute continuity and uniqueness of $\mu$ in this class.

\section{Notation and preliminaries}

Here we briefly list some notations we use throughout the paper, even if most of the symbols we use are standard.

$\begin{array}{ll}B(x, \rho) & \text { Ball in } \mathbb{R}^{N} \text { of centre } x \text { and radius } \rho \\ x y, \overline{x y} & \text { Closed segment in } \mathbb{R}^{N} \text { and its length } \\ D_{\mu} & \mu \text {-tangential gradient (see Sect. 2.1) } \\ \mathrm{W}_{\mu}^{1, p} & \text { Sobolev space with respect to a measure (see Sect. 2.1) } \\ \text { Dim } \mu & \text { Dimension of the measure } \mu \text { (see Sect. 2.2) } \\ \theta_{k}^{*}(\mu, x) & k \text {-upper density of } \mu \text { at } x \text { (see Sect. 2.2) } \\ d_{\mu}(x) & \text { Pointwise dimension of } \mu \text { at } x \text { (see Sect. 2.2) } \\ \mathcal{L} & \text { Lebesgue measure } \\ \mathcal{H}^{k} & k \text {-dimensional Hausdorff measure } \\ \mathcal{H}_{x y}^{1} & 1 \text {-dimensional Hausdorff measure on the segment } x y \\ \mu\llcorner B & \text { Restriction of the measure } \mu \text { to the set } B \\ \mathcal{B}(\Omega) & \text { Borel subsets of } \Omega \\ \operatorname{Lip}(\Omega) & 1-\text { Lipschitz functions on } \Omega \subseteq \mathbb{R}^{N} \\ \mathcal{M}(\Omega), \mathcal{M}^{+}(\Omega) & \text { Spaces of the measures and of the positive } \\ |\gamma| & \text { measures on } \Omega \\ \|\gamma\| & \text { Total variation of } \gamma \in \mathcal{M}(\Omega) \\ & \text { Norm of } \gamma \text { in the space } \mathcal{M}(\Omega), \text { or total mass, i.e. } \\ \omega_{k} & |\gamma|(\Omega) \\ & \text { If } k \text { is an integer, Lebesgue measure of the unit } \\ & \text { ball in } \mathbb{R}^{k}\end{array}$


Throughout this paper the ambient space will be $\Omega$, an open, bounded and convex subset of $\mathbb{R}^{N}, N \geq 2$ and its diameter will be denoted by $R$.

\subsection{Calculus of Variations with respect to a measure}

Here we introduce some notions from [4].

Given a positive measure $\mu \in \mathcal{M}^{+}(\Omega)$, we consider the space

$$
X_{\mu}:=\left\{\varphi \in L_{\mu}^{1}:-\operatorname{div}(\varphi \mu) \in \mathcal{M}(\Omega)\right\},
$$

in some sense $X_{\mu}$ is the space of tangent fields to $\mu$. Then we define the tangent space to $\mu$ for $\mu$-a.e. $x \in \Omega$ as:

$$
T_{\mu}(x):=\mu-e s s \bigcup\left\{\varphi(x): \varphi \in X_{\mu}\right\} .
$$

The $\mu$-essential union is defined as a $\mu$-measurable closed multi-function such that:

- $\varphi \in X_{\mu} \Longrightarrow \varphi(x) \in T_{\mu}(x)$ for $\mu$-a.a. $x \in \Omega$.

- Between all the multi-functions with the previous property the $\mu$-essential union is minimal with respect to the inclusion a.e. .

Properties and applications of this definition of tangent space to a measure have been explored in various paper, among them we address to [4], [5], [3], [13]. Once we have the notion of tangent space to $\mu$, it is natural to define the notion of $\mu$-tangential gradient of a function $u \in \mathcal{D}(\Omega)$ as:

$$
D_{\mu} u(x)=P_{\mu}(x, D u(x)) \quad \mu-\text { a.e. },
$$

where we denoted by $P_{\mu}(x, \cdot)$ the orthogonal projection on $T_{\mu}(x)$ (which is clearly a subspace). It can be shown that the operator $D_{\mu}(x)$ is closable in $L_{\mu}^{p}$ and this leads to a suitable definition of Sobolev space with respect to $\mu$ :

Definition 2.1. The Sobolev space $\mathrm{W}_{\mu}^{1, p}(\Omega)$ is the completion of $\mathcal{D}(\Omega)$ with respect to the norm:

$$
\|u\|_{1, p}:=\|u\|_{L_{\mu}^{p}}+\left\|D_{\mu} u\right\|_{L_{\mu}^{p}} .
$$

An important property is the following generalization of the integration by part formula, which holds for any $v \in \mathrm{W}_{\mu}^{1, p}(\Omega)$ and $\varphi \in X_{\mu}$ :

$$
\int D_{\mu} u \cdot \varphi d \mu=-\langle\operatorname{div}(\varphi \mu), u\rangle
$$

Using these notions one can obtain that if $\mathfrak{C}(\mu)>-\infty$ then $f \in\left(W_{\mu}^{1, p}\right)^{\prime}$ and the relaxed energy of (1.3) is given by:

$$
\bar{E}(\mu, u)=\frac{1}{2} \int_{\Omega}\left|D_{\mu} u\right|^{2} d \mu-\langle f, u\rangle \quad u \in \mathrm{W}_{\mu}^{1, p}(\Omega),
$$

where $\langle\cdot, \cdot\rangle$ is the $\left(\mathrm{W}_{\mu}^{1, p},\left(W_{\mu}^{1, p}\right)^{\prime}\right)$ duality. 


\subsection{Dimension of a measure}

In this section we introduce a notion of "dimension" for measures belonging to $\mathcal{M}^{+}(\Omega)$, which we will use later:

Definition 2.2. The dimension of $\mu \in \mathcal{M}^{+}(\Omega)$ is defined by

$$
\operatorname{Dim} \mu:=\sup \left\{k: \mu \ll \mathcal{H}^{k}\right\} \text {. }
$$

where $\mathcal{H}^{k}$ denotes the $k$-dimensional Hausdorff measure.

Notice that if $\mu$ is made of pieces of different dimensions then $\operatorname{Dim} \mu$ is the smallest of these.

In order to calculate the dimension of a measure it will be sometimes useful to give another representation of it and this will be done in Proposition 2.5. First we need to introduce the notion of $k$-upper density of $\mu$ at $x$ :

Definition 2.3. The $k$-upper density of $\mu$ at $x$ is defined by

$$
\theta_{k}^{*}(\mu, x):=\varlimsup_{\rho \rightarrow 0} \frac{\mu(B(x, \rho))}{\omega_{k} \rho^{k}} .
$$

A first useful result about $\theta_{k}^{*}$ is the following

Theorem 2.4. The following facts hold:

a) $\theta_{k}^{*}(\mu, x) \leq t \forall x \in B \in \mathcal{B}(\Omega) \Longrightarrow \mu\left\llcorner B \leq 2^{k} t \mathcal{H}^{k}\llcorner B\right.$;

b) $\theta_{k}^{*}(\mu, x) \geq t \forall x \in B \in \mathcal{B}(\Omega) \Longrightarrow \mu(B) \geq t \mathcal{H}^{k}(B)$;

c) $\theta_{k}^{*}(\mu, x)<+\infty$ for $\mathcal{H}^{k}-$ a.e. $x \in \Omega$.

Parts a) and b) are two particular cases from Theorem 3.2 in [19]; c) can be obtained immediately from $b$ ).

Thanks to Theorem 2.4 it is quite easy to obtain the following characterization of the dimension:

Proposition 2.5. Given $\mu \in \mathcal{M}^{+}(\Omega)$, the following three numbers are equal:

$$
\begin{aligned}
& \mathrm{D}_{1}=\sup \left\{k: \mu \ll \mathcal{H}^{k}\right\}=\operatorname{Dim} \mu ; \\
& \mathrm{D}_{2}=\inf \left\{k: \exists B \subseteq \Omega, \mu(B)>0, \mathcal{H}^{k}(B)=0\right\} ; \\
& \mathrm{D}_{3}=\sup \left\{k: \theta_{k}^{*}(\mu, x)<+\infty \text { for } \mu-\text { a.e. } x \in \Omega\right\} .
\end{aligned}
$$

Proof. Let us note that immediate consequences of the definitions are the following: $\mu \ll \mathcal{H}^{k}$ for all $k<\mathrm{D}_{1}, \exists B \subseteq \Omega$ such that $\mu(B)>0$ and $\mathcal{H}^{k}(B)=0$ for all $k>\mathrm{D}_{2}$ and $\theta_{k}^{*}(\mu, x)=0 \mu$-a.e. for all $k<\mathrm{D}_{3}$.

Step 1: $\mathrm{D}_{1} \geq \mathrm{D}_{2}$.

If $k<\mathrm{D}_{2}$, by definition $\mathcal{H}^{k}(B)=0$ implies $\mu(B)=0$; in other words, $\mu \ll \mathcal{H}^{k}$, and then $k \leq \mathrm{D}_{1}$.

Step 2: $\mathrm{D}_{2} \geq \mathrm{D}_{3}$.

Let $k>\mathrm{D}_{2}, B \subseteq \Omega$ a subset as in the definition and $B_{i}=$ $\left\{x \in B: \theta_{k}^{*}(\mu, x) \leq i\right\}$; part a) of Theorem 2.4 implies that $\mu\left(B_{i}\right)=0$ for all $i$, and then $\theta_{k}^{*}(\mu, x)=+\infty \mu$-a.e., then $k \geq \mathrm{D}_{3}$. 
Step 3: $\mathrm{D}_{3} \geq \mathrm{D}_{1}$.

Let $k<\mathrm{D}_{1}$, thanks to part c) of Theorem 2.4 we have $\theta_{k}^{*}(\mu, x)<+\infty \mathcal{H}^{k}-$ a.e., and then $\theta_{k}^{*}(\mu, x)<+\infty \mu$-a.e., because $\mu \ll \mathcal{H}^{k}$. This assures that $k<\mathrm{D}_{3}$.

Definition 2.6. The pointwise dimension of $\mu$ at $x$ is defined by

$$
d_{\mu}(x)=\sup \left\{k: \theta_{k}^{*}(\mu, x)<+\infty\right\} .
$$

Thanks to Proposition 2.5 the dimension of $\mu$ is the $\mu-e s \sin f$ of the pointwise dimensions of $\mu$.

We now prove some simple facts about the behavior of the dimension under the action of Lipschitz continuous functions, which we will need in Sect. 4.3 to show that no nontrivial upper estimates for the dimension of $\mu$ can be given.

Lemma 2.7. If $\mu \in \mathcal{M}^{+}(\Omega)$ and $\varphi: \Omega \rightarrow \mathbb{R}^{M}$ is a Lipschitz continuous function, then $\operatorname{Dim} \varphi_{\#} \mu \leq \operatorname{Dim} \mu$. Moreover, if $\varphi$ is bilipschitz then $\operatorname{Dim} \varphi_{\#} \mu=\operatorname{Dim} \mu$.

Proof. Using the definition of dimension of a measure we just need to prove that $\varphi_{\#} \mu \ll \mathcal{H}^{k} \Longrightarrow \mu \ll \mathcal{H}^{k}$. Then, let $\varphi_{\#} \mu \ll \mathcal{H}^{k}$ and let $A \in \mathcal{B}(\Omega)$ be a set such that $\mathcal{H}^{k}(A)=0$ : we have $\mu(A) \leq \mu\left(\varphi^{-1}(\varphi(A))=\varphi_{\#} \mu(\varphi(A))=0\right.$, where the last equality holds because $\mathcal{H}^{k}(\varphi(A))=0$. Finally, if $\varphi$ is bilipschitz, the same argument gives also the other inequality, since $\varphi_{\#}^{-1}\left(\varphi_{\#} \mu\right)=\mu$.

Lemma 2.8. Let $\mu \in \mathcal{M}^{+}(\Omega)$ and let $\varphi: \Omega \rightarrow \mathbb{R}^{M}$ be a Lipschitz map with the property that $\mu$-almost all of $\Omega$ can be covered by countable many Borel sets $A_{n}, n \in \mathbb{N}$, such that $\varphi$ is bilipschitz on each of the $A_{n}$. Then $\operatorname{Dim} \varphi_{\#} \mu=\operatorname{Dim} \mu$.

Proof. We just need to prove that $\operatorname{Dim} \varphi_{\#} \mu \geq \operatorname{Dim} \mu$, and this inequality will follow if we prove that $\mu \ll \mathcal{H}^{k} \Longrightarrow \varphi_{\#} \mu \ll \mathcal{H}^{k}$; let then $k$ be such that $\mu \ll \mathcal{H}^{k}$, and $A \in \mathcal{B}\left(\mathbb{R}^{M}\right)$ with $\mathcal{H}^{k}(A)=0$. Thanks to the previous lemma and to the assumptions, we have

$$
\varphi_{\#} \mu(A)=\mu\left(\varphi^{-1}(A)\right) \leq \sum_{n \in \mathbb{N}} \mu\left(\varphi^{-1}(A) \cap A_{n}\right)=0 .
$$

\section{Transport set and connections between the different problems}

In this section we will study the problem

$$
(W M K)= \begin{cases}-\operatorname{div}\left(\mu D_{\mu} u\right)=f & \text { on } \Omega \\ \left|D_{\mu} u\right|=1 & \mu-\text { a.e. }\end{cases}
$$

already introduced in Sect. 1.2. Besides the deep connections with the MongeKantorovich problem (see [3] and [1]), another interest of this problem is that, as shown in Theorem 3.8, the measure $\mu$ in a solution of (3.1), suitably rescaled, solves (1.4) and each solution of (1.4) can be obtained in this way. 
Let $u \in \operatorname{Lip}_{1}(\Omega)$ : it is usual (see [9], [20]) to associate to $u$ the so-called transport set as follows

$$
\begin{gathered}
T_{u}:=\left\{z \in \Omega: \exists x \in \operatorname{spt}\left(f^{+}\right) \text {s.t. } u(x)-|z-x|=u(z)\right. \text { and } \\
\left.\exists y \in \operatorname{spt}\left(f^{-}\right) \text {s.t. } u(y)+|y-z|=u(z)\right\} .
\end{gathered}
$$

If $x, y$ and $z$ are as in the definition of $T_{u}$ then they are aligned, in fact (we recall $u \in \operatorname{Lip}_{1}(\Omega)$ ) we have

$$
|x-y| \geq u(x)-u(y)=|x-z|+|z-y| .
$$

Moreover the closed segment $x y$ (which is often called transport ray with respect to $u$ ) is contained in the transport set.

Remark 3.1. $T_{u}$ is contained in the union of the segments joining $\operatorname{spt}\left(f^{+}\right)$and $\operatorname{spt}\left(f^{-}\right)$.

Remark 3.2. $T_{u}$ is a closed set.

In the next lemma we will use a test function which first appeared in a paper by Janfalk [16] and was also used by Evans and Gangbo in [9]. Let $v$ be defined as follows

$$
v(z)=\left\{\begin{array}{l}
u(z) \max _{w \in \operatorname{spt}(f)} \frac{\xi+u(w)}{\xi+u(z)+|w-z|} \text { if } u(z) \geq 0 \\
u(z) \max _{w \in \operatorname{spt}(f)} \frac{\xi-u(w)}{\xi-u(z)+|w-z|} \text { if } u(z) \leq 0,
\end{array}\right.
$$

where $\xi$ is a constant such that $\xi \pm u>0$ everywhere on $\Omega$.

Lemma 3.3. The function $v$ is Lipschitz continuous and satisfies the following properties:

a) $v=u$ on $\operatorname{spt}(f)$;

b) $|D v| \leq 1$ a.e.;

c) If $x \notin T_{u}$ then there exist a ball $B$ centered $x$ and a constant $\delta \in(0,1)$ such that $|D v| \leq 1-\delta$ on $B$.

The proof of this lemma is simple but not short, and can be found in [9], page 19-22.

Theorem 3.4. Let $(u, \mu)$ be a solution of (3.1) and let $T_{u}$ be the transport set related to $u$. Then $\operatorname{spt}(\mu) \subseteq T_{u}$.

Proof. Let $v$ be defined as in (3.2), $x_{0} \notin T_{u}$ and $B, \delta$ as in property c) of Lemma 3.3. Using the integration by parts formula and the estimate b) of Lemma 3.3 we obtain:

$$
\begin{aligned}
\int_{\Omega} v d f & =\int_{\Omega} D_{\mu} u \cdot D v d \mu \leq \int_{\Omega \backslash B}\left|D_{\mu} u\right||D v| d \mu+\int_{B}\left|D_{\mu} u\right||D v| d \mu \\
& \leq \int_{\Omega \backslash B}\left|D_{\mu} u\right| d \mu+(1-\delta) \int_{B}\left|D_{\mu} u\right| d \mu .
\end{aligned}
$$


Then, since property a) of Lemma 3.3 implies $\int_{\Omega} u d f=\int_{\Omega} v d f$, it follows

$$
\begin{aligned}
\delta \int_{B}\left|D_{\mu} u\right| d \mu & \leq \int_{\Omega}\left|D_{\mu} u\right| d \mu-\int_{\Omega} u d f \\
& =\int_{\Omega}\left|D_{\mu} u\right|^{2} d \mu-\int_{\Omega}\left|D_{\mu} u\right|^{2} d \mu=0
\end{aligned}
$$

also using (2.1). As $\int_{B}\left|D_{\mu} u\right| d \mu=\mu(B)$ we conclude that $\mu(B)=0$ and then $x_{0} \notin \operatorname{spt}(\mu)$.

Thanks to Remark 3.1 and Theorem 3.8, a consequence of the previous theorem is the following result concerning the region occupied by optimal distributions of the conductor, once given the heat sources.

Corollary 3.5. The optimal measures for problem (1.4) are supported in the union of the segments joining $\operatorname{spt}\left(f^{+}\right)$and $\operatorname{spt}\left(f^{-}\right)$.

Remark 3.6. The set of the segments joining $\operatorname{spt}\left(f^{+}\right)$and $\operatorname{spt}\left(f^{-}\right)$is clearly a subset of the convex envelope of $\operatorname{spt}(f)$, where $f=f^{+}-f^{-}$, and it can be strictly smaller. For example, if $\operatorname{spt}\left(f^{+}\right)$and $\operatorname{spt}\left(f^{-}\right)$are two concentric spherical surfaces, the first set is the annulus between the surfaces while the second is the whole sphere. It can also happen that the dimension of the first set is strictly smaller than that of the second, as in the next example.

Example 3.7. Let $A B C D$ be a square with sides of length $l$ and define $f^{+}=$ $\delta_{A}+\delta_{C}$ and $f^{-}=\delta_{B}+\delta_{D}$. In this case each transport set is contained in the boundary of the square, whose dimension is 1 , while the convex envelope of $s p t(f)$ is the whole square, whose dimension is 2 . Let us write now an explicit formula for the optimal shapes: denoted by $a=A B, b=B C, c=C D$ and $d=D A$ the sides of the square and fixed $0 \leq \alpha \leq 1$,

$$
\mu:=\alpha\left(\mathcal { H } ^ { 1 } \left\llcornera+\mathcal{H}^{1}\llcorner c)+(1-\alpha)\left(\mathcal { H } ^ { 1 } \left\llcornerb+\mathcal{H}^{1}\llcorner d)\right.\right.\right.\right.
$$

defines a solution of (3.1) together with any $u \in \operatorname{Lip}_{1}(\Omega)$ such that $u(A)=u(C)=$ $l$ and $u(B)=u(D)=0$. Vice versa for any solution $(u, \mu)$ of (3.1), the measure $\mu$ can be written as in (3.3) for a suitable $\alpha$. To prove what stated we remark that the admissible plans of transport have support contained in $\{(A, B),(A, D),(C, B)$, $(C, D)\}$, so it is easy to write explicitely each of them (note that they are all optimal) and then, thanks to the general formula (4.2), the optimal measures $\mu$.

Let us finally prove the connections between (1.4), (1.5), (1.7) and (3.1) with the following result, first given in [5]:

Theorem 3.8. Problem (1.4) has a solution and (1.6) holds. Moreover if $u$ and $\nu$ are optimal for (1.5) and (1.7) then $(u,|\nu|)$ solves (3.1) and, conversely, if $(u, \mu)$ solves (3.1) then $u$ and $\mu D_{\mu} u$ are optimal for (1.5) and (1.7) respectively. Finally, a measure $\mu$ solves (3.1) together with some $u \in \operatorname{Lip}_{1}(\Omega)$ if and only if $-m \mu / I(f)$ solves problem (1.4). 
Proof. Thanks to standard duality facts it is possible - even if not straigthforward - to prove that

$$
\begin{aligned}
& I(f)=\sup \left\{-\int d|\nu|: \nu \in \mathcal{M}\left(\Omega, \mathbb{R}^{N}\right),-\operatorname{div} \nu=f\right\}, \\
& \mathfrak{C}(\mu)=\sup \left\{-\frac{1}{2} \int|\sigma|^{2} d \mu: \sigma \in L_{\mu}^{2}\left(\Omega, \mathbb{R}^{N}\right),-\operatorname{div}(\sigma \mu)=f\right\}
\end{aligned}
$$

and that the extremals in (1.5), (1.7) and (3.5) are reached. Then we have

Step 1: $\mathcal{E}(f) \leq-I(f)^{2} /(2 m)$.

Let $\mu \in \mathcal{M}^{+}(\Omega)$ with $\int d \mu=m, \sigma$ such that $-\operatorname{div}(\sigma \mu)=f$ and $u \in \operatorname{Lip}_{1}(\Omega)$; then

$$
\langle f, u\rangle^{2}=\left(\int \sigma D u d \mu\right)^{2} \leq \int|\sigma|^{2} d \mu \int|D u|^{2} d \mu \leq m \int|\sigma|^{2} d \mu,
$$

which implies

$$
-\frac{1}{2} \int|\sigma|^{2} d \mu \leq-\frac{\langle f, u\rangle^{2}}{2 m}:
$$

thanks to (3.5), taking the sup in the left hand side and the inf in the right hand side we obtain the claimed inequality.

Step 2: Let $u$ and $\nu$ be optimal respectively for (1.5) and (1.7): thanks to (3.4) we have $-I(f)=\langle f, u\rangle=\int d|\nu|$. Define then $\mu=|\nu|$ and $\theta: \Omega \rightarrow \mathbb{R}^{N}$ such that $\nu=\theta \mu$ (and then $|\theta|=1 \mu$-a.e.). Using (2.1) we have then

$$
\int d \mu=\int d|\nu|=\langle f, u\rangle=\langle-\operatorname{div}(\theta \mu), u\rangle=\int D_{\mu} u \cdot \theta d \mu,
$$

which implies $\left|D_{\mu} u\right|=1 \mu$-a.e. and $\theta=D_{\mu} u$, then $(u, \mu)$ solves (3.1). Define now $\bar{\mu}=-m \mu / I(f)$ and $\bar{u}=-I(f) u / m$ : we have $\int d \bar{\mu}=m$ and $f=-\operatorname{div} \nu=$ $-\operatorname{div}\left(\bar{\mu} D_{\bar{\mu}} \bar{u}\right)$ so that, thanks to (3.5) and using (2.1),

$$
\mathfrak{C}(\bar{\mu}) \geq-\frac{1}{2} \int\left|D_{\bar{\mu}} \bar{u}\right|^{2} d \bar{\mu}=-\frac{1}{2}\langle f, \bar{u}\rangle=-\frac{I(f)^{2}}{2 m}:
$$

thanks to the first step this gives the optimality of $\bar{\mu}$ for problem (1.4) and the validity of (1.6).

Step 3: Let us take $(u, \mu)$ solution of (3.1) and define $\nu=\mu D_{\mu} u$, then $-\operatorname{div} \nu=f$ : we have

$$
I(f) \geq-\int d|\nu|=-\int d \mu=-\int\left|D_{\mu} u\right|^{2} d \mu=\langle-f, u\rangle \geq I(f),
$$

so that $I(f)=\langle-f, u\rangle=-\int d|\nu|$, which gives the stated optimality of $u$ and $\nu$ for (1.5) and (1.7). 
Step 4: Let $\mu$ be optimal for (1.4): then there exists $\sigma \in L_{\mu}^{2}\left(\Omega, \mathbb{R}^{N}\right)$ such that $-\operatorname{div}(\sigma \mu)=f$ and $\mathcal{E}(f)=\mathfrak{C}(\mu)=-1 / 2 \int|\sigma|^{2} d \mu$. Let us define $\nu=\sigma \mu$ and note that

$$
\begin{aligned}
\int d|\nu| & =\int|\sigma| d \mu \leq \sqrt{\int|\sigma|^{2} d \mu \sqrt{\int d \mu}} \\
& =\sqrt{-2 \mathcal{E}(f)} \sqrt{m}=\sqrt{\frac{I(f)^{2}}{m}} \sqrt{m}=-I(f),
\end{aligned}
$$

then $\nu$ is optimal for (1.7) and $|\sigma|$ must be constant, whence $|\sigma|=-I(f) / m$ $\mu$-a.e.: this implies that $-I(f) \mu / m$ solves (3.1) together with any $u$ optimal for (1.5).

\section{Dimension and summability of the transport density}

\subsection{Definition of the transport density}

In this section we will report formula (4.1), which was first introduced in [3], to write, starting from a solution $\gamma$ of (1.2), a measure $\nu$ which is extremal for (1.7); the measure $\mu=|\nu|$-which is given by formula (4.2)- is called, as we said in Sect. 1, transport density. Then, as proved in Theorem 3.8, $\mu$ solves (3.1) together with any $u$ extremal for (1.5) and, up to a rescaling constant, $\mu$ realizes the sup in (1.4). Depending on the point of view, then, this measure $\mu$ can be seen as a transport density for Monge-Kantorovich problem or as an optimal shape for the shape optimization problem. As seen in [1], [17] this measure $\mu$ is also related to an ODE version of the optimal transport problem introduced by Brenier and explained in [2] (see also [1]). Notice that $\mu$ will be defined starting from an optimal plan of transport $\gamma$, while the data of the problem are $f^{+}$and $f^{-}$: in fact, in general different optimal plans can generate different transport densities, as it happens in Example 3.7. However, in [1] it is proved that any optimal shape $\mu$ can be obtained by formula (4.2), starting from a suitable optimal plan $\gamma$, and that if at least one of the measures $f^{+}$and $f^{-}$is absolutely continuous then there is a unique transport density, which then can be found starting from any solution $\gamma$ of (1.2). In this section we will study the regularity properties of the measure $\mu$ in relation with the analogous properties of $f^{+}$and $f^{-}$. We will not use anything that comes from the particular choice of $\gamma$ : then the properties we will find are owned by any $\mu$ solving (3.1).

From now on we consider a fixed optimal plan of transport $\gamma$ from $f^{+}$to $f^{-}$, and in this section we will call transport ray each closed segment $x y$ such that $(x, y) \in \operatorname{spt}(\gamma)$; the relationship with the transport set and the transport rays defined in the previous section is that for each optimal plan of transport $\gamma$ there exists a 1 -Lipschitz function $u$ such that the transport rays associated to $\gamma$ are contained in $T_{u}$ and are transport rays also in the sense of Sect. 3 with respect to $u$. Moreover, given $x, y \in \Omega$, we denote by $\mathcal{H}_{x y}^{1}$ the one-dimensional Hausdorff measure on the segment $x y$. Finally we can define $\nu \in \mathcal{M}\left(\Omega, \mathbb{R}^{N}\right)$ as follows: 


$$
\langle\nu, \varphi\rangle:=\int_{\Omega \times \Omega}\left(\int_{\Omega} \varphi(z) \cdot \frac{x-y}{|x-y|} d \mathcal{H}_{x y}^{1}(z)\right) d \gamma(x, y)
$$

$\forall \varphi \in C_{0}\left(\Omega, \mathbb{R}^{N}\right)$. Let us prove now the extremality property of $\nu$ :

Proposition 4.1. The measure $\nu$ defined by (4.1) is extremal for (1.7).

Proof. First we need to verify that $-\operatorname{div} \nu=f$, and this holds since for any $\varphi \in \mathcal{D}(\Omega)$ we have

$$
\begin{aligned}
\langle-\operatorname{div} \nu, \varphi\rangle & =\langle\nu, D \varphi\rangle=\int_{\Omega \times \Omega} \int_{\Omega} D \varphi(z) \cdot \frac{x-y}{|x-y|} d \mathcal{H}_{x y}^{1}(z) d \gamma(x, y) \\
& =\int_{\Omega \times \Omega} \varphi(x)-\varphi(y) d \gamma(x, y) \\
& =\int_{\Omega} \varphi d f^{+}-\int_{\Omega} \varphi d f^{-}=\int_{\Omega} \varphi d f=\langle f, \varphi\rangle .
\end{aligned}
$$

Now, using a standard duality theorem for functional (1.2) due to Kantorovich, we know that

$$
\sup _{|D \varphi| \leq 1}\langle\varphi, f\rangle=\int_{\Omega \times \Omega}|y-x| d \gamma(x, y) .
$$

On the other hand, using (4.1) we obtain

$$
\int_{\Omega} d|\nu|=\int_{\Omega \times \Omega}|y-x| d \gamma(x, y)=\sup _{|D \varphi| \leq 1}\langle\varphi, f\rangle=-I(f),
$$

that thanks to (3.4) gives the assert.

We define now the transport density $\mu$ (which depends on $\gamma$ ) as the total variation of $\nu$. To begin, it is useful to write an explicit formula for $\mu$, which follows from (4.1):

$$
\langle\mu, \varphi\rangle:=\int_{\Omega \times \Omega}\left(\int_{\Omega} \varphi(z) d \mathcal{H}_{x y}^{1}(z)\right) d \gamma(x, y)
$$

In particular from (4.2) it is possible to write the measure $\mu$ of a set $A \in \mathcal{B}(\Omega)$ as

$$
\mu(A)=\int_{\Omega \times \Omega} l(x y \cap A) d \gamma(x, y),
$$

where $l(x y \cap A)=\mathcal{H}_{x y}^{1}(A)$ is the length of the intersection between the segment $x y$ and $A$. It is easy to note that if the mass is moved from $f^{+}$to $f^{-}$following the plan of transport $\gamma$, then $\mu(A)$ is the work done in the set $A$ : this is the reason why $\mu$ is called transport density. 


\subsection{Some technical lemmas}

In this section we report some technical results which we will need in Sects. 4.3 and 4.4. We will begin with two propositions, then we will give three geometric lemmas. A first property which follows directly from the definition of $\mu$ is the following:

Proposition 4.2. $\mu$-a.e. point in $\Omega$ is contained in the interior of some transport ray.

Proof. This is a consequence of the definition of $\mu$ : let $S$ be the subset of the points of $\Omega$ which are not contained in the interior of a transport ray. For every $(x, y) \in \operatorname{spt}(\gamma)$ we have $x y \cap S \subseteq\{x, y\}$ and then $l(x y \cap S)=0$ which, from (4.3), gives the claimed assertion.

Let us recall now a simple but useful property of the optimal plans of transport:

Proposition 4.3. If $\gamma \in \mathcal{M}^{+}(\Omega \times \Omega)$ is an optimal plan of transport from $\pi_{\#}^{1} \gamma$ to $\pi_{\#}^{2} \gamma$ and $\tau \in \mathcal{M}^{+}(\Omega \times \Omega)$ is a measure such that $\tau \leq \gamma($ i.e. $\tau(A) \leq \gamma(A)$ for each $A \in \mathcal{B}(\Omega)$ ), then $\tau$ is an optimal plan from $\pi_{\#}^{1} \tau$ to $\pi_{\#}^{2} \tau$. In particular $\gamma\llcorner(B \times \Omega)$, where $B$ is any Borel subset of $\Omega$, is optimal.

Proof. By contradiction, if $\tau$ were not optimal, it would exist $\tilde{\tau} \in \mathcal{M}^{+}(\Omega \times \Omega)$ with the same marginals as $\tau$ but less total work. In that case $\gamma-\tau+\tilde{\tau}$ would be a positive measure with the same marginals as $\gamma$ and less total work, which contradicts the optimality of $\gamma$.

The above proposition will be very useful in the proofs of the next results, when it will be convenient to divide $\Omega$ in subsets with assigned properties.

The next three lemmas consider a point $z$ contained in the interior of a transport ray $x y$ (which holds for $\mu-$ a.a. point, thanks to Proposition 4.2) and give estimates about the location of the extreme points of the other rays which meet an open ball centered at $z$ and of sufficiently small radius: in particular in Lemma 4.4 we find a region to which at least one of the extrems belongs, in Lemma 4.5 we find a region to which both the extrems belong, but the estimate degenerates when $z$ get closer to $x$ or $y$, in Lemma 4.6 we find a region to which the first extrem of the ray belong, but under the assumption that $z$ is not contained in the support of $f^{-}$. These lemmas will play a key role in the proofs of all the next results of this paper.

To prove these lemmas we will only use property (1.9); it will be convenient to reduce the possible configurations of the set of points we will use by moving them suitably; obviously we are allowed only to move the points in such a way that (1.9) continues to hold. Then, given $(x, y)$ and $\left(x^{\prime}, y^{\prime}\right)$ in $s p t(\gamma)$, we will call admissible transformations all the changes of the points such that the quantity

$$
\overline{x y}+\overline{x^{\prime} y^{\prime}}-\overline{x y^{\prime}}-\overline{x^{\prime} y}
$$

decreases, which clearly maintain the validity of (1.9): for example, thanks to the triangular inequality it is easily seen that moving $x$ to $y$ or $y$ to $x$ on the line $x y$ is admissible, and the same holds for $x^{\prime}, y^{\prime}$ and $x^{\prime} y^{\prime}$. 
Lemma 4.4. If $z$ is an interior point of a transport ray $x y, \varepsilon$ is sufficiently small and another ray $x^{\prime} y^{\prime}$ of length less than $\alpha \overline{x y}$ (with $\alpha \geq 1$ ) intersects $B(z, \varepsilon)$, then either $x^{\prime}$ or $y^{\prime}$ belongs to the cylinder $C_{\varepsilon}$ with axis $x y$ and radius $6 \alpha \varepsilon$.

Proof. Let us assume $\varepsilon \ll 1$ and that there exists a transport ray $x^{\prime} y^{\prime}$ of length less than $\alpha \overline{x y}$ that intersects $B(z, \varepsilon)$ but neither $x^{\prime}$ nor $y^{\prime}$ is in the cilinder of axis $x y$ and radius $6 \alpha \varepsilon$ : in order to prove the lemma we must show that this leads to a contradiction.

By symmetry, we can assume that $0<a \leq b \leq R$, where $a=\overline{x z}, b=\overline{x y}$ and $R$ is the diameter of $\Omega$ : let us move $x^{\prime}$ closer to $y^{\prime}$ moving it on the line $x^{\prime} y^{\prime}$ (which is admissible) until the distance between $x^{\prime}$ and the line $x y$ is $5 \alpha \varepsilon$. We fix now coordinates $\left\{c_{1}, \ldots, c_{N}\right\}$ such that $z$ is the origin, the segment $x y$ is on the first axis (with $c_{1}(x)<0, c_{1}(y)>0$ ), $x^{\prime}$ is on the plane $\left\{c_{3}=\cdots=c_{N}=0\right\}$ with $c_{2}\left(x^{\prime}\right)<0$ (and then $c_{2}\left(x^{\prime}\right)=-5 \alpha \varepsilon$ ) and $y^{\prime}$ is in the space $\left\{c_{4}=\cdots=c_{N}=0\right\}$ with $c_{3}\left(y^{\prime}\right) \geq 0$. Since the distance between $y^{\prime}$ and the line $x y$ is greater then $6 \alpha \varepsilon$, $x^{\prime} y^{\prime}$ intersects $B(z, \varepsilon)$ and $c_{3}\left(x^{\prime}\right)=c_{3}(z)=0$, from Pitagora's theorem it follows that $c_{2}\left(y^{\prime}\right)>5 \alpha \varepsilon$ : bring then $y^{\prime}$ closer to $x^{\prime}$ (moving it on the line $x^{\prime} y^{\prime}$ ) until $c_{2}\left(y^{\prime}\right)=5 \alpha \varepsilon$. The situation is illustrated in Fig. 1 .

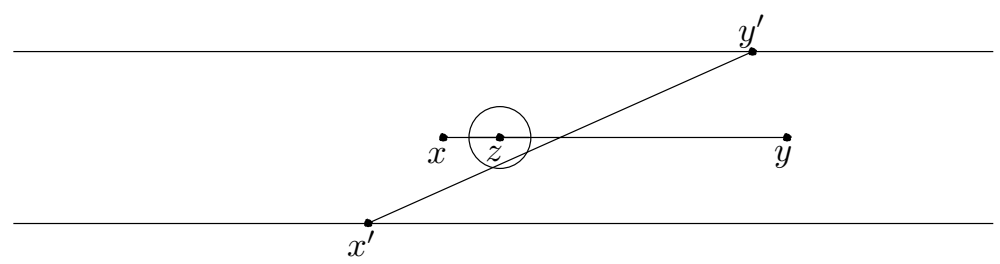

Fig. 1. Geometry of lemma 4.4

Let us define now $\delta=c_{1}\left(y^{\prime}\right)$ and $l=c_{1}\left(y^{\prime}\right)-c_{1}\left(x^{\prime}\right)$. We can assume $l \geq 0$ : otherwise, applying to $x^{\prime}$ and $y^{\prime}$ the simmetry across the hyperplane $\left\{c_{1}=\lambda\right\}$ with $\lambda=0, c_{1}\left(y^{\prime}\right)$ or $c_{1}\left(x^{\prime}\right)$ respectively if $c_{1}\left(y^{\prime}\right) \leq 0 \leq c_{1}\left(x^{\prime}\right), 0 \leq c_{1}\left(y^{\prime}\right) \leq c_{1}\left(x^{\prime}\right)$ or $c_{1}\left(y^{\prime}\right) \leq c_{1}\left(x^{\prime}\right) \leq 0$ changes the sign of $l$ and is an admissible transformation, because $\overline{x y}$ and $\overline{x^{\prime} y^{\prime}}$ remain equal while $\overline{x y^{\prime}}$ and $\overline{x^{\prime} y}$ increase. Moreover we have $l \gg \varepsilon$ : otherwise, using (1.9) and the fact that it would be $\overline{x^{\prime} y^{\prime}} \geq \sqrt{l^{2}+(10 \alpha \varepsilon)^{2}}$ and $\overline{x y^{\prime}}+\overline{x^{\prime} y}=a+b+l+(5 \alpha \varepsilon)^{2}(1 / 2 a+1 / 2 b)+o\left(\varepsilon^{2}\right)$, one easily find $l \geq 4 a b /(a+b) \gg \varepsilon$. We have then $l \gg \varepsilon$ and, as a consequence, $\delta \gg \varepsilon$ : the coordinates of the points are now

$$
\begin{aligned}
& x \equiv(-a, 0,0) \quad y \quad \equiv(b, 0,0) \\
& x^{\prime} \equiv(\delta-l,-5 \alpha \varepsilon, 0) \quad y^{\prime} \equiv(\delta, 5 \alpha \varepsilon, h \varepsilon),
\end{aligned}
$$

writing only the first three coordinates because all the points are in the space $\left\{c_{4}=\cdots=c_{N}=0\right\}$. The facts that $x^{\prime} y^{\prime}$ intersects $B(z, \varepsilon), c_{3}\left(x^{\prime}\right)=c_{3}(z)=0$, $b \geq a>0$ and $l \leq \overline{x^{\prime} y^{\prime}} \leq \alpha \overline{x y}$ imply

$$
0 \leq h<2.5 \quad \delta+\varepsilon \geq \frac{5 \alpha-1}{10 \alpha} l \quad \delta-\varepsilon \leq \frac{5 \alpha+1}{10 \alpha} l \quad b \geq \frac{\overline{x y}}{2} \geq \frac{l}{2 \alpha} .
$$


Let us write now the lengths of the segments:

$$
\begin{array}{ll}
\overline{x y}=a+b, & \overline{x^{\prime} y}=b+l-\delta+\frac{25 \alpha^{2}+o(1)}{2(b+l-\delta)} \varepsilon^{2} \\
\overline{x^{\prime} y^{\prime}}=l+\frac{100 \alpha^{2}+h^{2}+o(1)}{2 l} \varepsilon^{2}, & \overline{x y^{\prime}}=a+\delta+\frac{25 \alpha^{2}+h^{2}+o(1)}{2(a+\delta)} \varepsilon^{2} .
\end{array}
$$

The inequality (1.9) gives $\overline{x y}+\overline{x^{\prime} y^{\prime}} \leq \overline{x y^{\prime}}+\overline{x^{\prime} y}$ and this, thanks to (4.5) and using (4.4), implies

$$
\begin{aligned}
\frac{100 \alpha^{2}+h^{2}}{2 l} \leq & \frac{25 \alpha^{2}}{2(b+l-\delta)}+\frac{25 \alpha^{2}+h^{2}}{2(a+\delta)}+o\left(\varepsilon^{2}\right) \Longrightarrow \\
\frac{100 \alpha^{2}+h^{2}}{l} \leq & \frac{25 \alpha^{2}}{l /(2 \alpha)+l-((5 \alpha+1) / 10 \alpha) l} \\
& +\frac{25 \alpha^{2}+h^{2}}{((5 \alpha-1) / 10 \alpha) l} \Longrightarrow \\
750 \alpha^{3}-400 \alpha^{2} \leq & h^{2}\left(25 \alpha^{2}+25 \alpha+4\right) \Longrightarrow \\
750 \alpha^{3}-400 \alpha^{2} \leq & 6.25\left(25 \alpha^{2}+25 \alpha+4\right),
\end{aligned}
$$

which is easily seen to be false for each $\alpha \geq 1$.

Lemma 4.5. If $z$ is an interior point of a transport ray $x y, a=\min \{\overline{x z}, \overline{z y}\}, \varepsilon$ is sufficiently small and another transport ray $x^{\prime} y^{\prime}$ intersects $B(z, \varepsilon)$, then both $x^{\prime}$ and $y^{\prime}$ belong to the cylinder $C_{\varepsilon}$ with axis $x y$ and radius $5 R \varepsilon / a$.

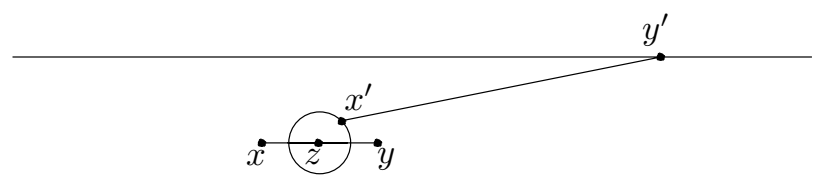

Fig. 2. Geometry of Lemma 4.5

Proof. Possibly moving $x$ closer to $y$ or $y$ closer to $x$ on the line $x y$, which we know to be an admissible transformation, we can assume that $\overline{x y}=2 a$ and $z$ is the middle point of $x y$; let us also assume that $y^{\prime}$ does not belong to the cylinder and show that this leads to a contradiction. Since $y^{\prime}$ does not belong to the ball $B(z, \varepsilon)$ but $x^{\prime} y^{\prime}$ intersects the ball, we can move $x^{\prime}$ in the direction of $y^{\prime}$ until it crosses $B(z, \varepsilon)$ and exits from it, and this is admissible.

We can assume now that $x, y, x^{\prime}$ and $y^{\prime}$ are on a plane: in fact, moving $y^{\prime}$ to the point of least distance from $x^{\prime}$ remaining on the $(N-2)$-dimensional sphere of the points whose distances from $x$ and $y$ are fixed is admissible (because in this way $\overline{x y}, \overline{x y^{\prime}}$ and $\overline{x^{\prime} y}$ remain fixed, while $\overline{x^{\prime} y^{\prime}}$ decreases) and causes all the point to be on a same plane whitout changing the fact that $y^{\prime} \notin C_{\varepsilon}$. Finally we can move 
$y^{\prime}$ closer to $x^{\prime}$ until it reaches $C_{\varepsilon}$, and the situation is illustrated in Fig. 2. If we fix coordinates in the obvious way (as we did in the previous lemma), the points are

$$
\begin{array}{rlrl}
x & \equiv(-a, 0) & y & \equiv(a, 0) \\
x^{\prime} \equiv(\delta \varepsilon, h \varepsilon) & y^{\prime} \equiv(\delta \varepsilon+l, 5 R \varepsilon / a)
\end{array}
$$

and we know that

$$
a \leq R \quad l \leq R \quad-1 \leq \delta \leq 1 \quad 0 \leq h \leq 1:
$$

writing the lengths of the segments and using (1.9) and (4.6) exactly as we did in the proof of Lemma 4.4, we find a contradiction, and then the desired inequality.

Lemma 4.6. If $z$ is an interior point of a transport ray $x y, d\left(z, \operatorname{spt}\left(f^{-}\right)\right)=\delta>0$, $\varepsilon$ is sufficiently small and another ray $x^{\prime} y^{\prime}$ intersects $B(z, \varepsilon)$, then $x^{\prime}$ belongs to the cylinder $C_{\varepsilon}$ of axis $x y$ and radius $6 R \varepsilon / \delta$, where $R$ is the diameter of $\Omega$.

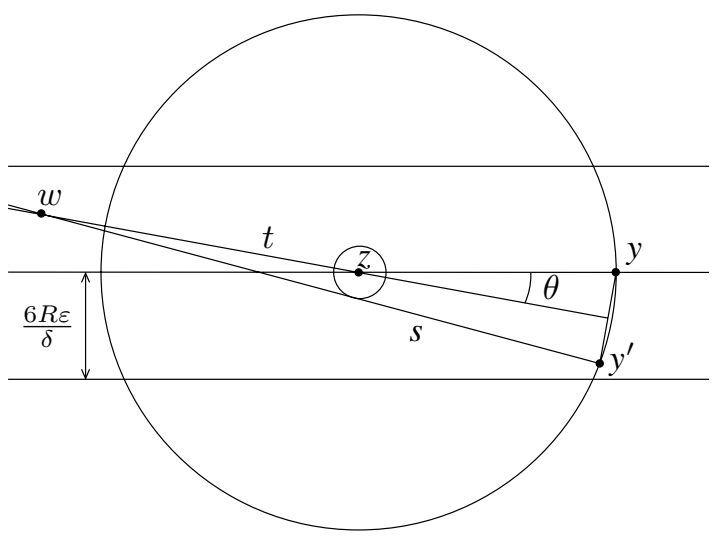

Fig. 3. Geometry of Lemma 4.6

Proof. Let us begin with the admissible transformations of bringing $y$ and $y^{\prime}$ closer to $x$ and $x^{\prime}$ (on the lines $x y$ and $x^{\prime} y^{\prime}$ ) until they are at a distance $\delta$ from $z$, which is possible if $\varepsilon \leq \delta$. If $x^{\prime}$ belongs to $B(z, \varepsilon)$ we have nothing to prove, otherwise $\overline{x^{\prime} y^{\prime}} \geq \delta$-since $\overline{z y^{\prime}}=\delta$ and $x^{\prime} y^{\prime}$ intersects $B(z, \varepsilon)$ - and then, applying Lemma 4.4, we know that either $x^{\prime}$ or $y^{\prime}$ belong to the cylinder of axis $x y$ and ray $6 \varepsilon \overline{x^{\prime} y^{\prime}} / \overline{x y} \leq$ $6 R \varepsilon / \delta$. If $x^{\prime}$ belongs to that cylinder there is nothing to prove, then let us assume that $y^{\prime}$ belongs to it and move $x$ closer to $y$ until $x \equiv z$. Finally we fix a system of coordinates such that $z$ is the origin, $y \equiv(\delta, 0, \ldots, 0), y^{\prime}$ belongs to the plane $\left\{c_{3}=\cdots c_{N}=0\right\}$ with $c_{2}\left(y^{\prime}\right) \leq 0$ and $x^{\prime}$ belongs to the space $\left\{c_{4}=\cdots c_{N}=0\right\}$. Since $c_{3}\left(y^{\prime}\right)=c_{3}(z)=0$ and $x^{\prime} y^{\prime}$ intersects $B(z, \varepsilon)$, it follows by similitude that $\left|c_{3}\left(x^{\prime}\right)\right| \leq R \varepsilon /(\delta-\varepsilon) \leq 2 R \varepsilon / \delta$ if $\varepsilon$ is sufficiently small. Moreover the fact that 
$c_{2}\left(y^{\prime}\right) \leq 0$ implies, by a similar argument, that $c_{2}\left(x^{\prime}\right) \geq-2 R \varepsilon / \delta$ : it will be then sufficient to prove that $c_{2}\left(x^{\prime}\right) \leq 5 R \varepsilon / \delta$.

To this aim let us note that $y^{\prime}$ belongs to $C_{\varepsilon}$ and $\overline{z y^{\prime}}=\delta$, and then if $\varepsilon \ll 1$ we have $\left|c_{1}\left(y^{\prime}\right)\right| \approx \delta$; then we can assume that $c_{1}\left(y^{\prime}\right)>0$ : in fact, if $c_{1}\left(y^{\prime}\right) \leq 0$ then $c_{1}\left(y^{\prime}\right) \approx-\delta$ and then either $c_{1}\left(x^{\prime}\right) \leq 0$, and then easily $c_{2}\left(x^{\prime}\right)<5 R \varepsilon / \delta$ if $\varepsilon \ll 1$, or $c_{1}\left(x^{\prime}\right)>0$, and then apply to $x^{\prime}$ and $y^{\prime}$ the simmetry across the hyperplane $\left\{c_{1}=0\right\}$ is admissible and leads us to a situation in which $c_{1}\left(y^{\prime}\right)>0$.

If $c_{2}\left(y^{\prime}\right) \geq-3 \varepsilon$ a similitude argument similar to that we already used implies $c_{2}\left(x^{\prime}\right) \leq 5 R \varepsilon / \delta$; if $c_{2}\left(y^{\prime}\right)<-3 \varepsilon$, let us consider the situation, illustrated by Fig. 3: denoting by $x_{2}$ the projection of $x^{\prime}$ on the plane $\left\{c_{3}=\cdots=c_{N}=0\right\}$, since $x^{\prime} y^{\prime}$ intersects $B(z, \varepsilon)$, the point $x_{2}$ is over the line $s$, and since $\overline{x^{\prime} y^{\prime}} \leq \overline{x^{\prime} y}$ (from (1.9) recalling that $\overline{x y}=\overline{x y^{\prime}}$ ) it is under the line $t$, the axis of the segment $y y^{\prime}$ : then $c_{2}\left(x^{\prime}\right)=c_{2}\left(x_{2}\right) \leq c_{2}(w)$. With some elementary (and a little boring) calculations we can then write the equations of the lines $s$ and $t$ and using the fact that, thanks to our assumptions, $-c_{2}\left(y^{\prime}\right)=\delta \sin (2 \theta) \in[3 \varepsilon, 6 R \varepsilon / \delta]$, we find $c_{2}(w) \leq 4 \varepsilon \leq 4 R \varepsilon / \delta$ if $\varepsilon \ll 1$, that concludes the proof.

\subsection{Dimension of the optimal measure}

This section is entirely devoted to discuss estimates for the dimension (in the sense of Sect. 2.2) of $\mu$ : we will prove Theorem 4.7, which gives a lower estimate (sharp thanks to an example), and then we will show that no upper estimate is possible. Let us begin with the lower estimate, which follows by a careful analysis from Lemma 4.5 .

Theorem 4.7. $\operatorname{Dim} \mu \geq \max \left\{\operatorname{Dim} f^{+}, \operatorname{Dim} f^{-}, 1\right\}$.

Proof. Step 1: Dim $\mu \geq 1$.

From (4.3) it follows that for any $x \in \Omega$ and $\rho>0$ we have $\mu(B(x, \rho)) \leq 2 \rho\|\gamma\|$ which implies, from Definition 2.3, that $\theta_{1}^{*}(\mu, x) \leq\|\gamma\|$ for any $x$. Then using Proposition 2.5 we have the claim.

Let us assume now that $\operatorname{Dim} f^{+} \geq \operatorname{Dim} f^{-}$(we can do it by the symmetry of the problem) and denote $k=\operatorname{Dim} f^{+}$. We have to prove that $\operatorname{Dim} \mu \geq k$.

Step 2: Assume for the moment that the following hypothesis holds:

$$
\exists K: \forall x \in \Omega, \forall \rho>0 f^{+}(B(x, \rho)) \leq K \rho^{k} ;
$$

we will get rid of it in the next step.

If $z$ is contained in the interior of some transport ray $x y$ and $a=\min \{\overline{x z}, \overline{z y}\}$, let $C_{\rho}$ be the cylinder or axis $x y$ and radius $5 R \rho / a$, which clearly can be covered by $M(z) / \rho$ balls of radius $\rho$ : this, thanks to (4.7), assures that

$$
f^{+}\left(C_{\rho}\right) \leq \frac{M(z)}{\rho} K \rho^{k} \leq M(z) K \rho^{k-1} .
$$

Then, if $\rho$ is sufficiently small, using Lemma 4.5 we have

$$
\begin{aligned}
\mu(B(z, \rho)) & =\int_{C_{\rho} \times C_{\rho}} l(x y \cap B(z, \rho)) d \gamma(x, y) \leq 2 \rho \int_{C_{\rho} \times C_{\rho}} d \gamma(x, y) \\
& \leq 2 \rho f^{+}\left(C_{\rho}\right) \leq 2 M(z) K \rho^{k} .
\end{aligned}
$$


We then infer that $\theta_{k}^{*}(\mu, x)<+\infty$ for ever $z \in \Omega$ which is contained in the interior of some transport ray, and then from Propositions 2.5 and 4.2 it follows that $\operatorname{Dim} \mu \geq k$. The theorem is then proved under the additional assumption (4.7). Step 3: General case.

Fix $\varepsilon>0$ and define for any $m \in \mathbb{N}$

$$
\Omega_{m, \varepsilon}=\left\{x \in \Omega: \forall \rho>0, f^{+}(B(x, \rho)) \leq m \rho^{k-\varepsilon}\right\} .
$$

Since $k-\varepsilon<\operatorname{Dim} f^{+}$, we have $\theta_{k-\varepsilon}^{*}\left(f^{+}, x\right)=0 f^{+}$-a.e., as noted in the beginning of the proof of Proposition 2.5, and then

$$
\sup _{\rho>0} \frac{f^{+}(B(x, \rho))}{\rho^{k-\varepsilon}}<+\infty \quad \text { for } f^{+} \text {-a.e. } x:
$$

then, varying $m, \Omega_{m, \varepsilon}$ is an increasing sequence of subsets that fills $\Omega$ up to an $f^{+}$-negligible set and then, as $m \rightarrow+\infty, f^{+}\left\llcorner\Omega_{m, \varepsilon} \longrightarrow f^{+}\right.$in the strong convergence of $\mathcal{M}^{+}(\Omega)$. Defining $\gamma_{m, \varepsilon}=\gamma\left\llcorner\left(\Omega_{m, \varepsilon} \times \Omega\right)\right.$ and recalling that $\pi_{\#}^{1} \gamma=f^{+}$we infer that $\gamma_{m, \varepsilon} \longrightarrow \gamma$. Let us define now $\mu_{m, \varepsilon}$ from $\gamma_{m, \varepsilon}$ by (4.2). It is clear from the definition that $\mu_{m, \varepsilon} \longrightarrow \mu$, and thanks to Proposition 4.3 we can apply the result of step 2 to $\Omega_{m, \varepsilon}$ to obtain $\operatorname{Dim} \mu_{m, \varepsilon} \geq k-\varepsilon$ : this, thanks to the definition of dimension and to the convergence of $\mu_{m, \varepsilon}$, implies Dim $\mu \geq k-\varepsilon$. Finally the generality of $\varepsilon$ completes the proof.

The next example shows that the lower estimate given in Theorems 4.7 is sharp.

Example 4.8. Let $S_{1}$ and $S_{2}$ be two disjoint segments of length $l$ lying on the same line, and let $f^{+}$and $f^{-}$be the restrictions of the measure $\mathcal{H}^{1}$ to $S_{1}$ and $S_{2}$ respectively. Clearly an optimal transport is given by the translation, then it is simple to calculate $\mu$, which is the measure on the line which density is $l$ between the segments, goes to 0 linearly on the segments and is 0 out, and which is then one-dimensional.

On the other hand, it is not possible to give a non-trivial estimate from above of the dimension, as the following example shows:

Example 4.9. Let $x_{1}, x_{2}, B_{1}$ and $B_{2}$ be respectively two points and two balls of unit volume in $\Omega$, and let $f^{+}=\delta_{x_{1}}+\mathcal{L}\left\llcorner B_{1}\right.$ and $f^{-}=\delta_{x_{2}}+\mathcal{L}\left\llcorner B_{2}\right.$ : if $x_{1}$ is near to $B_{2}, x_{2}$ is near to $B_{1}$ and $x_{1}, B_{2}$ are far from $x_{2}, B_{1}$, there is a unique optimal plan of transport, which distributes on $B_{2}$ the mass of $x_{1}$ and concentrates on $x_{2}$ the mass of $B_{1}$. In this case the dimension of $\mu$ is easily seen to be $N$, the maximum possible, while the dimensions of $f^{+}$and $f^{-}$is 0 , the least possible.

The lack of control in the previous example is due to the fact that the pointwise dimensions of $f^{+}$and $f^{-}$are not constant. However, even if the pointwise dimensions are constant it is not possible to give an upper estimate of $\operatorname{Dim}\left(f^{+} \otimes f^{-}\right)$ in terms of $\operatorname{Dim} f^{+}$and $\operatorname{Dim} f^{-}$(on this topic see: 3.2.23, 2.10.45, 2.10.29 of [11] and theorem 5.11 of [10]). As in the next example we show how to construct an optimal measure $\mu$ whose dimension is equal to $\operatorname{Dim}\left(f^{+} \otimes f^{-}\right)+1$, it is not possible to give a non trivial estimate from above of $\operatorname{Dim} \mu$ even if the pointwise dimensions of $f^{+}$and $f^{-}$are constant. 
Example 4.10. Let $p, k$ be two positive integers such that $p+1+k \leq N, S$ be the unit sphere of the subspace of $\mathbb{R}^{N}$ given by the first $(p+1)$ coordinates and $B$ be the unit ball of the subspace given by the coordinates from the $(p+2)-t h$ to the $(p+1+k)-t h$. Let $f^{+}$and $f^{-}$be two probability measures on $S$ and $B$ respectively. By the symmetry of the problem it follows that any plan of transport is optimal. If we take, for example, $\gamma=f^{+} \otimes f^{-}$, then we remark that $\mu$ is the push forward of $f^{+} \otimes f^{-} \otimes \mathcal{L}^{1}\left\llcorner[0,1]\right.$ through the map $\varphi: S \times B \times[0,1] \rightarrow \mathbb{R}^{N}$ defined by $(x, y, t) \rightarrow t x+(1-t) y$. Applying Lemma 2.8 taking $A_{n}=S \times B \times(1 / n, 1-1 / n)$, we obtain $\operatorname{Dim} \mu=\operatorname{Dim}\left(f^{+} \otimes f^{-} \otimes \mathcal{L}^{1}\llcorner[0,1])=\operatorname{Dim}\left(f^{+} \otimes f^{-}\right)+1\right.$.

\subsection{Summability of the optimal measure}

In this section we will investigate the summability of $\mu$. Let us first remark that for a measure the property of being absolutely continuous is a bit stronger than having dimension $N$, which is obviously the maximal possible dimension in $\Omega$. From Theorem 4.7 we already know that if $f^{+}$has dimension $N$, then also the dimension of $\mu$ is $N$. The first result we will give will be a little step forward and it represents the connection between the study of the dimension and that of the summability of $\mu$ :

Theorem 4.11. If at least one between $f^{+}$and $f^{-}$is absolutely continuous with respect to the Lebesgue measure, then $\mu$ is absolutely continuous with respect to the same measure.

Proof. Step 1: Let us first assume that $f^{+} \in L^{\infty}$.

If $z$ is contained in the interior of some transport ray, a simple application of Lemma 4.5 (similar to the one made in second step of Theorem 4.7) gives a constant $K(z)$ such that $\mu(B(z, \varepsilon)) \leq K(z)\left\|f^{+}\right\|_{L^{\infty}} \varepsilon^{N}$ for $\varepsilon$ sufficiently small, and then $\theta_{N}^{*}(\mu, z)<+\infty$. Since this holds for each $z$ contained in the interior of some transport ray, from Proposition 4.2 we have

$$
\theta_{N}^{*}(\mu, z)<+\infty \text { for } \mu \text {-a.e. } z \text {. }
$$

If now $\mathcal{L}(B)=0$ and we define $B_{m}=\left\{z \in B: \theta_{N}^{*}(\mu, z) \leq m\right\}$, part a) of Theorem 2.4 implies

$$
\mu\left(B_{m}\right) \leq 2^{N} m \mathcal{H}^{N}\left(B_{m}\right) \leq 2^{N} m \mathcal{H}^{N}(B)=0,
$$

which together with (4.8) assures $\mu(B)=0$. Then $\mu \in L^{1}$.

Step 2: General case.

Let $\Omega_{m}=\left\{x \in \Omega: f^{+}(x) \leq m\right\}, \gamma_{m}=\gamma\left\llcorner\left(\Omega_{m} \times \Omega\right)\right.$ and let $\mu_{m}$ be defined from $\gamma_{m}$ using (4.2). Thanks to Proposition 4.3 and to the first step we can infer that $\mu_{m} \in L^{1}$. Arguing as in the proof of Theorem 4.7, $\gamma_{m} \longrightarrow \gamma$ in $\mathcal{M}^{+}(\Omega \times \Omega)$, and then $\mu_{m} \longrightarrow \mu$ in $\mathcal{M}^{+}(\Omega)$, which gives $\mu \in L^{1}$ in the general case too.

We can generalize this last result, studying what happens if $f^{+}$(or $f^{-}$) belongs to $L^{q}$ for some $q>1$. The previous theorem shows that $\mu \in L^{1}$, but we can also prove that $\mu$ belongs to $L^{p}$ for some $p>1$. Let us begin with the case $q=+\infty$, the general result will then follow. In the sequel we will denote by $\alpha^{\prime}$ the conjugate exponent of $\alpha$, i.e. $1 / \alpha+1 / \alpha^{\prime}=1$. 
Lemma 4.12. If $f^{+} \in L^{\infty}$ then $\mu \in L^{p}$ for all $p<(2 N)^{\prime}$. More precisely

$$
\|\mu\|_{L^{p}} \leq K\left\|f^{+}\right\|_{L^{\infty}}^{1 / p^{\prime}}\left\|f^{+}\right\|_{L^{1}}^{1 / p}
$$

where $K$ depends only on $R, N$ and $p$.

Proof. Given $(x, y) \in \operatorname{spt}(\gamma)$, let $y_{\sigma}$ be the point on the segment $x y$ such that

$\overline{y y_{\sigma}}=\sigma$, or $y_{\sigma}=x$ if $\overline{x y}<\sigma$. Let us define the measure $\mu_{r}$ as follows:

$$
\left\langle\mu_{r}, \varphi\right\rangle:=\int_{\Omega \times \Omega}\left(\int_{\Omega} \varphi(z) d \mathcal{H}_{y_{r} y_{2 r}}^{1}(z)\right) d \gamma(x, y) .
$$

Thanks to (4.2), it is clear that $\mu=\mu_{R}+\mu_{R / 2}+\mu_{R / 4}+\ldots$, where $R$ is the diameter of $\Omega$. From Theorem 4.11 we know that $\mu \in L^{1}$, and then $\mu_{r} \in L^{1}$ because $\mu_{r} \ll \mu$. It is useful to write the measure $\mu_{r}$ of a set $A$, which from (4.9) is

$$
\mu_{r}(A)=\int_{\Omega \times \Omega} l\left(y_{r} y_{2 r} \cap A\right) d \gamma(x, y),
$$

where $l(x y \cap A)$ is understood as in (4.3).

Step 1: Let us fix $r>0$ and begin with a very particular case: we assume that $\operatorname{spt}\left(f^{-}\right) \subseteq Q$, where $Q$ is an hypercube of side $\lambda$ such that $\lambda \sqrt{N} \leq r / 2$ (note that $\lambda \sqrt{N}$ is the diameter of $Q$ ). From the definition, it follows that $\operatorname{spt}\left(\mu_{r}\right)$ is contained in an annulus $S$ with radii $r-\lambda \sqrt{N} / 2$ and $2 r+\lambda \sqrt{N} / 2$ centered at the center of the hypercube, furthermore $l\left(y_{r} y_{2 r} \cap S\right) \leq r$ for all $(x, y)$ and then

$$
\left\|\mu_{r}\right\|_{L^{1}}=\mu_{r}(S) \leq r f^{-}(Q) .
$$

On the other hand, the hypothesis $\lambda \sqrt{N} \leq r / 2$ assures that $d(S, Q) \geq r / 2$ and then from Lemma 4.6 (arguing as in the proofs of Theorems 4.7 and 4.11) it follows that

$$
\left\|\mu_{r}\right\|_{L^{\infty}} \leq 2 \omega_{N-1}\left(\frac{6 R}{r / 2}\right)^{N-1} R\left\|f^{+}\right\|_{L^{\infty}}=\frac{C\left\|f^{+}\right\|_{L^{\infty}}}{r^{N-1}}
$$

It is well known that if $\varphi \in L^{1} \cap L^{\infty}$ then $\varphi \in L^{p}$ and

$$
\|\varphi\|_{L^{p}} \leq\|\varphi\|_{L^{1}}^{1 / p}\|\varphi\|_{L^{\infty}}^{1 / p^{\prime}}
$$

then from (4.10) and (4.11) we infer that

$$
\left\|\mu_{r}\right\|_{L^{p}} \leq\left(r f^{-}(Q)\right)^{1 / p}\left(\frac{C\left\|f^{+}\right\|_{L^{\infty}}}{r^{N-1}}\right)^{1 / p^{\prime}} \leq \frac{K\left\|f^{+}\right\|_{L^{\infty}}^{1 / p^{\prime}} f^{-}(Q)^{1 / p}}{r^{N-1-N / p}} .
$$

Step 2: Let us cover $\operatorname{spt}\left(f^{-}\right)$with $N(r)$ disjoint hypercubes $Q_{i}$ with sides as in step 1 and define $\gamma_{i}=\gamma L\left(\Omega \times Q_{i}\right)$. From Proposition 4.3 it follows that $\gamma_{i}$ is an optimal plan of transport from $f_{i}^{+}=\pi_{\#}^{1} \gamma_{i}$ to $f_{i}^{-}=\pi_{\#}^{2} \gamma_{i}=f^{-}\left\llcorner Q_{i}\right.$. Define $\mu_{r, i}$ from $\gamma_{i}$ using (4.9): from the first step and the fact that $\left\|f_{i}^{+}\right\|_{L^{\infty}} \leq\left\|f^{+}\right\|_{L^{\infty}}$ we obtain that $\mu_{r}=\mu_{r, 1}+\mu_{r, 2}+\cdots+\mu_{r, N(r)}$ and

$$
\left\|\mu_{r, i}\right\|_{L^{p}} \leq \frac{K\left\|f^{+}\right\|_{\infty}^{1 / p^{\prime}} f^{-}\left(Q_{i}\right)^{1 / p}}{r^{N-1-N / p}}
$$


Applying the inequality between the arithmetic mean and the $p$-th mean, integrating, extracting the $p$-th root and using the fact that $f^{+}(\Omega)=f^{-}(\Omega)$, we obtain that

$$
\left\|\mu_{r}\right\|_{L^{p}} \leq N(r)^{1 / p^{\prime}} \frac{K\left\|f^{+}\right\|_{\infty}^{1 / p^{\prime}}}{r^{N-1-N / p}} f^{+}(\Omega)^{1 / p} .
$$

We recall $\mu=\mu_{R}+\mu_{R / 2}+\mu_{R / 4}+\ldots$ and we remark that, up to a geometric constant, $N(r) \leq r^{-N}$, then it suffices to show that

$$
\sum_{i \in \mathbb{N}}\left(\frac{R}{2^{i}}\right)^{-N+1+N / p-N / p^{\prime}}<+\infty
$$

a simple calculation assures that (4.12) holds if and only if $p<(2 N)^{\prime}$, and this concludes the proof.

We now derive the general result:

Theorem 4.13. If $f^{+} \in L^{q}$ then $\mu \in L^{p}$ for all $p<(2 N)^{\prime} \wedge(1+(q-1) / 2)$.

Proof. Let $p<(2 N)^{\prime} \wedge(1+(q-1) / 2)$ and let us write $\Omega=\bigcup_{i} \Omega_{i}$ up to a

$f^{+}$-negligible set, where $\Omega_{i}=\left\{x: i-1 \leq f^{+}(x)<i\right\}$. As in the previous lemma we can define $\gamma_{i}=\gamma L\left(\Omega_{i} \times \Omega\right)$ and $\mu_{i}$ from $\gamma_{i}$ using (4.2). Observe that, thanks to Proposition $4.3, \gamma_{i}$ is an optimal plan of transport. As $p<(2 N)^{\prime}$, we can then apply Lemma 4.12 obtaining

$$
\left\|\mu_{i}\right\|_{L^{p}} \leq K i^{1 / p^{\prime}} f^{+}\left(\Omega_{i}\right)^{1 / p} \leq K i^{1 / p^{\prime}}\left(i \cdot\left|\Omega_{i}\right|\right)^{1 / p} \leq K i\left|\Omega_{i}\right|^{1 / p} .
$$

Denoted $\lambda_{i}=\left|\Omega_{i}\right|$, it is sufficient to prove that $\sum_{i} i \lambda_{i}^{1 / p}<+\infty$. Let us define

$$
\rho_{i}:=\left\{\begin{array}{l}
\lambda_{i} \text { if } \lambda_{i} \geq i^{p^{\prime}(1-q)} \\
0 \text { if } \lambda_{i}<i^{p^{\prime}(1-q)}
\end{array} \quad \sigma_{i}:=\left\{\begin{array}{l}
0 \text { if } \lambda_{i} \geq i^{p^{\prime}(1-q)} \\
\lambda_{i} \text { if } \lambda_{i}<i^{p^{\prime}(1-q)}
\end{array}:\right.\right.
$$

it is clear that $\sum_{i} i \lambda_{i}^{1 / p}=\sum_{i} i \rho_{i}^{1 / p}+\sum_{i} i \sigma_{i}^{1 / p}$. Since $p<1+(q-1) / 2$, the fact that $\sigma_{i}<i^{p^{\prime}(1-q)}$ implies, by a simple calculation, that $\sum_{i} i \sigma_{i}^{1 / p}<+\infty$. Moreover $f^{+} \in L^{q}$ easily implies $\sum i^{q} \lambda_{i}<+\infty$ and then, since by definition $i \rho_{i}^{1 / p} \leq i^{q} \lambda_{i}, \sum i \rho_{i}^{1 / p}<+\infty$, that gives the assert.

Now, a natural question that arises is the following: which is the greatest $p$ such that $f^{+} \in L^{q} \Longrightarrow \mu \in L^{p}$ ? The previous theorem gives a lower bound for this number, but it is easy to give also an upper bound. In fact, thanks to the next example, this number cannot be greater then $q$ :

Example 4.14. In $\mathbb{R}^{2}$ let $R_{1}=[-2,-1] \times[0,1]$ and $R_{2}=[1,2] \times[0,1]$. Moreover let $g:[0,1] \rightarrow \mathbb{R}$ be an absolutely continuous function, consider $a^{+}: R_{1} \rightarrow \mathbb{R}$ and $a^{-}: R_{2} \rightarrow \mathbb{R}$ defined by $a^{+}(x, y)=a^{-}(-x, y)=g(y)$ and define $f^{+}=$ $a^{+} \mathcal{H}^{2}\left\llcorner R_{1}\right.$ and $f^{-}=a^{-} \mathcal{H}^{2}\left\llcorner R_{2}\right.$. Thanks to the symmetry of the problem the horizontal translation of $f^{+}$on $f^{-}$is an optimal transport, then using formula (4.2) 
we can explicitely write the measure $\mu$ obtaining that it is absolutely continuous and supported in $[-2,2] \times[0,1]$ with density given by

$$
v(x, y)= \begin{cases}g(y)(x+2) & \text { if } x \in[-2,-1] \\ g(y) & \text { if } x \in[-1,1] \\ g(y)(2-x) & \text { if } x \in[1,2]\end{cases}
$$

Then the summability of $\mu$ is exactly the same of $f^{+}$and $f^{-}$.

Another fact is that $f^{+} \in L^{\infty}$ does not imply $\mu \in L^{\infty}$ (and in a similar way $f^{+} \in L^{p}$ does not imply $\mu \in L^{p}$ ), as the next example shows:

Example 4.15. Let $f^{-}$be the Dirac mass in 0 and $f^{+}$be the restriction of the Lebesgue measure to an annulus centered in 0 and of unitary volume, then $f^{+} \in$ $L^{\infty}$. Obviously $\gamma=f^{+} \otimes f^{-}$, which brings all the mass of $f^{+}$in 0 , is the unique plan of transport and then is optimal. It is then easy, using (4.3), to evaluate $\mu(x)$ for each Lebesgue point $x$ for $\mu$, which we already know, thanks to Theorem 4.11, to be absolutely continuous: it follows $\mu(x) \propto|x|^{1-N}$ for $x$ in the interior of the annulus, and then $\mu \notin L^{\infty}$; more precisely, $\mu \in L^{p}$ if and only if $p<N /(N-1)$.

We want finally study what happens when both $f^{+}, f^{-} \in L^{q}$ : we already know, by Example 4.14, that $\mu$ need not to belong to any $L^{p}$ with $p>q$, but we can expect some result stronger than Theorem 4.13, due to the summability of both the measures $f^{+}$and $f^{-}$. We start with the following technical lemma:

Lemma 4.16. If $g=\sum_{i \in X}\left|g_{i}\right|, g_{i} \in L^{\infty} \cap L^{1}, X \subseteq \mathbb{Z}$ and $1 \leq p<+\infty$, then

$$
\int g^{p} d x \leq \sum_{i \in X} \frac{\left\|g_{i}\right\|_{L^{1}}}{\left\|g_{i}\right\|_{L^{\infty}}}\left(\sum_{j \leq i}\left\|g_{i}\right\|_{L^{\infty}}\right)^{p} .
$$

Proof. Let us first assume that $X$ is finite. Then, in the subset of $\left(L^{\infty} \cap L^{1}\right)^{X}$ for which $\left\|g_{i}\right\|_{L^{1}}$ and $\left\|g_{i}\right\|_{L^{\infty}}$ are fixed, the maximum of $\int g^{p}$ is attained in the case $g_{i}=\left\|g_{i}\right\|_{L^{\infty}} \chi_{A_{i}}$, where $\mathcal{L}\left(A_{i}\right)\left\|g_{i}\right\|_{L^{\infty}}=\left\|g_{i}\right\|_{L^{1}}$ and the sets $A_{i}$ intersect as much as possible. The formula is then straightforward is $X$ is composed by two elements, and an easy induction argument implies the validity in general. Since all the terms are positive, a passage to the limit gives the assert also for $X$ infinite.

We can prove now that if both $f^{+}$and $f^{-}$are in $L^{\infty}$ then also $\mu \in L^{\infty}$.

Proposition 4.17. If both $f^{+}$and $f^{-}$are in $L^{\infty}$, then $\mu \in L^{\infty}$. More precisely, $\|\mu\|_{L^{\infty}} \leq 2 R \omega_{N-1} 12^{N-1}\left(\left\|f^{+}\right\|_{L^{\infty}}+\left\|f^{-}\right\|_{L^{\infty}}\right)$.

Proof. Let us define $\Lambda_{i}=\left\{(x, y) \in \Omega \times \Omega: R / 2^{i+1}<\overline{x y} \leq R / 2^{i}\right\}$, where $R$ is the diameter of $\Omega$, and $\mu_{i}, i \in \mathbb{N}$, by

$$
\mu_{i}(A):=\int_{\Lambda_{i}} l(x y \cap A) d \gamma(x, y) .
$$


Recalling (4.3) it is clear that $\mu=\sum_{i \in \mathbb{N}} \mu_{i}$. We already know that $\mu \in L^{1}$, and then $\mu_{i} \in L^{1}$ for each $i \in \mathbb{N}$ : let us give then the last definition,

$$
\begin{aligned}
\Omega_{i}:= & \left\{z \in \Omega: \mu_{i}(z)>0, \nexists(x, y) \in \Lambda_{i} \cap \operatorname{spt}(\gamma)\right. \\
& \text { s.t. } z \text { is in the interior of } x y\} .
\end{aligned}
$$

From (4.13) it follows that $\mu_{i}\left(\Omega_{i}\right)=0$, because for each $(x, y) \in \Lambda_{i}$ we have $x y \cap \Omega_{i} \subseteq\{x, y\}$ and then $l\left(x y \cap \Omega_{i}\right)=0$. Then $\mathcal{L}\left(\Omega_{i}\right)=0$, and this implies $\mathcal{L}\left(\bigcup_{i} \Omega_{i}\right)=0$ and then $\mu\left(\bigcup_{i} \Omega_{i}\right)=0$.

Let $z \notin \bigcup_{i} \Omega_{i}$ be a Lebesgue point for each of the $\mu_{i}$ such that $\mu(z)=$ $\sum_{i} \mu_{i}(z)>0$, and let $j$ be the least integer such that $\mu_{j}(z)>0$. Since $z \notin \Omega_{j}$, there exists a transport ray $u v$ such that $z$ is in the interior of $u v$ and $R / 2^{j+1}<$ $\overline{u v} \leq R / 2^{j}$, and then if $\overline{x y} \leq R / 2^{j}$ we have $\overline{x y} \leq 2 \overline{u v}$. Let us then denote by $C_{r}$ the cylinder of axis $u v$ and radius $12 r$ : thanks to Lemma 4.4, if $r \ll 1$

$$
\begin{aligned}
\sum_{i=j}^{\infty} \mu_{i}(B(z, r)) & =\int_{\overline{x y} \leq R / 2^{j}} l(B(z, r) \cap x y) d \gamma(x, y) \\
& \leq 2 r\left(\int_{C_{r} \times \Omega} d \gamma(x, y)+\int_{\Omega \times C_{r}} d \gamma(x, y)\right) \\
& \leq 2 r\left(f^{+}\left(C_{r}\right)+f^{-}\left(C_{r}\right)\right) .
\end{aligned}
$$

Since the volume of the cone $C_{r}$ is less than $R \omega_{N-1}(12 r)^{N-1}$,

$$
\sum_{i=j}^{\infty} \mu_{i}(B(z, r)) \leq 2 r R \omega_{N-1}(12 r)^{N-1}\left(\left\|f^{+}\right\|_{L^{\infty}}+\left\|f^{-}\right\|_{L^{\infty}}\right),
$$

which gives

$$
\mu(z)=\sum_{i=j}^{\infty} \mu_{i}(z) \leq 2 R \omega_{N-1} 12^{N-1}\left(\left\|f^{+}\right\|_{L^{\infty}}+\left\|f^{-}\right\|_{L^{\infty}}\right):
$$

since this inequality holds for $\mu$-a.e. point $z$, this completes the proof.

Once we solved the problem for $f^{+}, f^{-} \in L^{\infty}$, we can prove the general assert, which shows the situation when both $f^{+}, f^{-} \in L^{q}$ :

Theorem 4.18. If both $f^{+}, f^{-} \in L^{q}$, then $\mu \in L^{p}$ for $1 \leq p<q$.

Proof. Let us define $\Omega_{i}^{+}, \Omega_{i}^{-}, \mu_{i j}$ and $\lambda_{i j}$ with $i, j \in \mathbb{N}$ as follows

$$
\begin{aligned}
\Omega_{0}^{ \pm} & :=\left\{x \in \Omega: f^{ \pm}(x) \leq 1\right\}, \quad \Omega_{i}^{ \pm}:=\left\{x \in \Omega: 2^{i-1}<f^{ \pm}(x) \leq 2^{i}\right\}, \\
\mu_{i j} & :=\int_{\Omega_{i}^{+} \times \Omega_{j}^{-}} l(x y \cap A) d \gamma(x, y), \quad \lambda_{i j}:=\gamma\left(\Omega_{i}^{+} \times \Omega_{j}^{-}\right):
\end{aligned}
$$

then $\mu=\sum_{i, j} \mu_{i j}$, it is easy to give the bound

$$
\left\|f^{ \pm}\right\|_{L^{q}}^{q} \geq \sum_{i \geq 1} 2^{(i-1) q} \mathcal{L}\left(\Omega_{i}^{ \pm}\right) \geq \sum_{i \geq 1} \frac{2^{i(q-1)} f^{ \pm}\left(\Omega_{i}^{ \pm}\right)}{2^{q}}
$$


and we have also $\sum_{j} \lambda_{i j}=f^{+}\left(\Omega_{i}^{+}\right)$and $\sum_{i} \lambda_{i j}=f^{-}\left(\Omega_{j}^{-}\right)$. From the definition it follows that $\left\|\mu_{i j}\right\|_{L^{1}} \leq R \lambda_{i j}$ and, since $\gamma L\left(\Omega_{i} \times \Omega_{j}\right)$ is an optimal plan of transport for the problem given by his marginals, thanks to Proposition 4.17 we have $\left\|\mu_{i j}\right\|_{L^{\infty}} \leq K\left(2^{i}+2^{j}\right)$. Thanks to these estimates, to give an upper bound for the $L^{p}$ norm of $\mu$ we can make use of Lemma 4.16 with $X=\mathbb{N}$ after given an isomorphism between $\mathbb{N}$ and $\mathbb{N} \times \mathbb{N}$, or equivalently an order to $\mathbb{N} \times \mathbb{N}$. We do it by setting $(m, n)<(i, j)$ if $m \vee n<i \vee j$, or if $m \vee n=i \vee j$ and $m \wedge n<i \wedge j$, or if $m=j, n=i$ and $m<n$. Then Lemma 4.16 gives

$$
\|\mu\|_{L^{p}}^{p} \leq \sum_{i, j} \frac{R \lambda_{i j}}{K\left(2^{i}+2^{j}\right)}\left(\sum_{(m, n) \leq(i, j)} K\left(2^{m}+2^{n}\right)\right)^{p} .
$$

But if $i \geq j$ then

$$
\sum_{(m, n) \leq(i, j)} 2^{m}+2^{n} \leq 2(i+1)\left(1+2+4+\cdots+2^{i}\right) \leq(i+1) 2^{i+2},
$$

and then from (4.15) we have

$$
\begin{aligned}
\|\mu\|_{L^{p}}^{p} \leq & R K^{p-1}\left(\sum_{i \geq j} \frac{\lambda_{i j}\left((i+1) 2^{i+2}\right)^{p}}{2^{i}}+\sum_{i \leq j} \frac{\lambda_{i j}\left((j+1) 2^{j+2}\right)^{p}}{2^{j}}\right) \\
\leq & 4^{p} R K^{p-1}\left(\sum_{i \geq j} \lambda_{i j}(i+1)^{p} 2^{i(p-1)}+\sum_{i \leq j} \lambda_{i j}(j+1)^{p} 2^{j(p-1)}\right) \\
\leq & \widetilde{K}\left(\sum_{i}\left((i+1)^{p} 2^{i(p-1)} \sum_{j} \lambda_{i j}\right)\right. \\
& \left.+\sum_{j}\left((j+1)^{p} 2^{j(p-1)} \sum_{i} \lambda_{i j}\right)\right) \\
\leq & \widetilde{K}\left(\sum_{i}(i+1)^{p} 2^{i(p-1)} f^{+}\left(\Omega_{i}^{+}\right)\right. \\
& \left.+\sum_{j}(j+1)^{p} 2^{j(p-1)} f^{-}\left(\Omega_{j}^{-}\right)\right) .
\end{aligned}
$$

By (4.14) and the fact that $p<q$, this proves the assert.

Remark 4.19. At the moment, we do not know if a better interpolation allows, from Proposition 4.17, to prove Theorem 4.18 even with $p=q$. In fact, we do not know any example whit $f^{+}, f^{-} \in L^{p}$ and $\mu \notin L^{p}$.

The last result can be strengthened if the supports of $f^{+}$and $f^{-}$are disjointed: in this case we can prove that if $f^{+}$and $f^{-}$are in $L^{p}$ then $\mu$ is also in $L^{p}$. This hypothesis of disjointeness plays an important role in many other situations, even because it is needed to use PDE tools: this is why, for example, it is assumed in [9], [15] and [8]. 
Theorem 4.20. If both $f^{+}$and $f^{-}$are in $L^{p}$ and $d\left(\operatorname{spt}\left(f^{+}\right), \operatorname{spt}\left(f^{-}\right)\right)=\delta>0$, then $\mu \in L^{p}$. More precisely,

$$
\|\mu\|_{L^{p}} \leq \frac{K\left(\left\|f^{+}\right\|_{L^{p}}+\left\|f^{-}\right\|_{L^{p}}\right)}{\delta^{(N-1) / p^{\prime}}}
$$

where $K$ depends only on $R, N$ and $p$.

Proof. Let us define $B^{ \pm}=\left\{x \in \Omega: d\left(x, \operatorname{spt}\left(f^{ \pm}\right) \geq \delta / 2\right\}\right.$ : since $\delta$ is the distance between the supports of $f^{+}$and $f^{-}, \Omega=B^{+} \cup B^{-}$, then we will study the summability of $\mu^{ \pm}=\mu\left\llcorner B^{ \pm}\right.$. Let us first define, for $i \in \mathbb{Z}, \Omega_{i}=\left\{x: 2^{i}<\right.$ $\left.f^{+}(x) \leq 2^{i+1}\right\}, f_{i}^{+}=f^{+}\left\llcorner\Omega_{i}, \gamma_{i}=\gamma\left\llcorner\left(\Omega_{i} \times \Omega\right), \tilde{\mu}_{i}\right.\right.$ from $\gamma_{i}$ using (4.2) and $\mu_{i}=\tilde{\mu}_{i}\left\llcorner B^{-}:\right.$it is clear that $\mu^{-}=\sum_{i \in \mathbb{Z}} \mu_{i}$. Moreover thanks to the definition of $f_{i}^{+}$we have $\left\|f_{i}^{+}\right\|_{L^{1}} \leq 2^{i+1} \mathcal{L}\left(\Omega_{i}\right)$ and $\left\|f_{i}^{+}\right\|_{L^{p}}^{p} \geq 2^{i p} \mathcal{L}\left(\Omega_{i}\right)$, and then $\left\|f_{i}^{+}\right\|_{L^{p}}^{p} \geq$ $2^{i p-i-1}\left\|f_{i}^{+}\right\|_{L^{1}}$; since $f^{+}=\sum_{i \in \mathbb{Z}} f_{i}^{+}$and the supports of $f_{i}^{+}$are disjointed, it follows

$$
\left\|f^{+}\right\|_{L^{p}}^{p} \geq \frac{1}{2} \sum_{i \in \mathbb{Z}} 2^{i(p-1)}\left\|f_{i}^{+}\right\|_{L^{1}}
$$

Thanks to the definition of $\mu_{i}$, the $L^{1}$-norm of $\mu_{i}$ is less then $R\left\|f_{i}^{+}\right\|_{L^{1}}$; moreover, using Lemma 4.6 we know that if $x \in B^{-}$and $\varepsilon$ is sufficiently small, then

$$
\mu_{i}(B(x, \varepsilon)) \leq 2 \varepsilon f_{i}^{+}\left(C_{\varepsilon}\right) \leq 2 \varepsilon 2^{i+1} R \omega_{N-1}\left(\frac{6 R \varepsilon}{\delta / 2}\right)^{N-1}:
$$

summarizing, we have found the following estimates for $\mu_{i}$

$$
\left\|\mu_{i}\right\|_{L^{1}} \leq R\left\|f_{i}^{+}\right\|_{L^{1}} \quad\left\|\mu_{i}\right\|_{L^{\infty}} \leq \frac{\omega_{N-1} 6^{N-1} R^{N} 2^{i+2}}{(\delta / 2)^{N-1}} .
$$

Thanks to Lemma 4.16 with $X=\mathbb{Z}$, this gives

$$
\begin{aligned}
\int\left(\mu^{-}\right)^{p} & \leq \sum_{i \in \mathbb{Z}} \frac{\left\|\mu_{i}\right\|_{L^{1}}}{\left\|\mu_{i}\right\|_{L^{\infty}}}\left(\sum_{j=-\infty}^{i}\left\|\mu_{i}\right\|_{L^{\infty}}\right)^{p} \\
& \leq R^{1+N(p-1)}(\delta / 2)^{(N-1)(1-p)} \omega_{N-1}^{p-1} 6^{(N-1)(p-1)} \\
& \times \sum_{i \in \mathbb{Z}} 2^{(i+3) p-(i+2)}\left\|f_{i}^{+}\right\|_{L^{1}} \\
& \leq R^{1+N(p-1)}(\delta / 2)^{(N-1)(1-p)} \omega_{N-1}^{p-1} 6^{(N-1)(p-1)} 2^{3 p-1}\left\|f^{+}\right\|_{L^{p}}^{p},
\end{aligned}
$$

using also (4.16). To estimate the $L^{p}$ norm of $\mu^{+}$, clearly, we can make exactly the same calculation above changing all the "+" with "-" and vice versa and then, since $\mu \leq \mu^{+}+\mu^{-}$, we can conclude the proof with the estimate

$$
\|\mu\|_{L^{p}} \leq \frac{2^{3-1 / p} R^{1+(N-1) / p^{\prime}} \omega_{N-1}^{1 / p^{\prime}} 12^{(N-1) / p^{\prime}}}{\delta^{(N-1) / p^{\prime}}}\left(\left\|f^{+}\right\|_{L^{p}}+\left\|f^{-}\right\|_{L^{p}}\right) .
$$


Acknowledgements. The authors wish to thank Luigi Ambrosio (S.N.S. Pisa) and Giuseppe Buttazzo (University of Pisa) for many useful conversations during the preparation of this paper. The authors are partially supported by the Italian GNAFA/INDAM through the project Problemi di Monge-Kantorovich e Strutture Geometriche Deboli.

\section{References}

1. L. Ambrosio, Lecture Notes on Optimal Transportation Problems; lectures given in Madeira (Portugal), 2-9 July 2000, preprint.

2. J.-D. Benamou, Y. Brenier, A computational fluid mechanics solution to the MongeKantorovich mass transfer problem, Numer. Math., 84 (2000), 375-393.

3. G. Bouchitté, G. Buttazzo, Characterization of optimal shapes and masses through Monge-Kantorovich Equation, J. Eur. Math. Soc. (JEMS) 3, 139-168.

4. G. Bouchitté, G. Buttazzo, P. Seppecher, Energy with Respect to a Measure and Applications to Low Dimensional Structures., Calc.Var., 5 (1997), 37-54.

5. G. Bouchitté, G. Buttazzo, P. Seppecher, Shape optimization solutions via MongeKantorovich equation, C.R. Acad. Sci. Paris, 324-I (1997), 1185-1191.

6. L.A. Caffarelli, M. Feldman, R. McCann, Constructing optimal maps in Monge's transport problem as a limit of strictly convex costs, to appear in J. Amer. Math. Soc.

7. I. Ekeland, R. Temam, Analyse Convexe et Problèmes Variationnels, Dunod GauthierVillars (1974).

8. L.C. Evans, Partial Differential Equations and Monge-Kantorovich Mass Transfer, Current Developments in Mathematics, forthcoming.

9. L.C. Evans, W. Gangbo, Differential Equations Methods for the Monge-Kantorovich Mass Transfer Problem, Mem. Amer.Math.Soc., Vol. 137 (1999).

10. K.J. Falconer, The Geometry of Fractal Sets, Cambridge University Press, Cambridge (1985).

11. H. Federer, Geometric Measure Theory, Grundlehren der Math. Wiss., vol. 153, Springer-Verlag, Berlin (1969).

12. M. Feldman, R.J. McCann, Uniqueness and transport density in Monge's mass transportation problem, Calc. Var. DOI 10.1007/s005260100119.

13. I. Fragalà, C. Mantegazza, On some notion of tangent space to a measure, Proc. Roy. Soc. Edimburgh, 129A (1999), 331-342.

14. W.Gangbo, The Monge mass transfer problem and its applications, Caffarelli, Luis A. (ed.) et al., Monge Ampere equation: applications to geometry and optimization. Providence, RI: American Mathematical Society. Contemp. Math., 226, 79-104 (1999). MSC 1991

15. W. Gangbo, R.J. McCann, The geometry of optimal transportation, Acta Math., 177 (1996), 113-161.

16. U. Janfalk, On certain problems concerning the $p$-Laplace operator, Linkøping Studies in Science and Technology, dissertation 326, Linkøping University, Sweden, 1993.

17. A. Pratelli, Regolarità delle misure ottimali nel problema di trasporto di MongeKantorovich, Degree thesis, University of Pisa, Italy, 2000.

18. S.T. Rachev, L. Ruschendorf, Mass Transportation Problems, Springer-Verlag (1998).

19. L. Simon, Lectures on Geometric Measure Theory, Proceedings of the Centre for Mathematical Analysis, Australian National University, Vol. 3 (1983).

20. V.N. Sudakov, Geometric problems in the Theory of Infinite-Dimensional Probability Distributions, Proc. of the Steklov Institue of Mathematics, 141 (1979).

21. N.S. Trudinger, X.-J. Wang, On the Monge Mass Transfer Problem, Calc. Var. 13 (2001), 19-31. 